\title{
Diagnosing Circumstellar Debris Disks
}

\author{
Joseph M. Hahn \\ Space Science Institute \\ 10500 Loring Drive \\ Austin, TX, 78750 \\ jhahn@spacescience.org \\ 512-291-2255
}

\author{
Review draft version 2 \\ Submitted for publication in the \\ Astrophysical Journal on April 21, 2010 \\ Revised June 16, 2010 \\ Accepted June 21, 2010
}




\begin{abstract}
A numerical model of a circumstellar debris disk is developed and applied to observations of the circumstellar dust orbiting $\beta$ Pictoris. The model accounts for the rates at which dust is produced by collisions among unseen planetesimals, and the rate at which dust grains are destroyed due to collisions. The model also accounts for the effects of radiation pressure, which is the dominant perturbation on the disk's smaller but abundant dust grains. Solving the resulting system of rate equations then provides the dust abundances versus grain size and over time. Those solutions also provide the dust grains' collisional lifetime versus grain size, and the debris disk's optical depth and surface brightness versus distance from the star. Comparison to observations then yields estimates of the unseen planetesimal disk's radius, and the rate at which the disk sheds mass due to planetesimal grinding. The model can also be used to measure or else constrain the dust grain's physical and optical properties, such as the dust grains' strength, their light scattering asymmetry parameter, and the grains' efficiency of light scattering $Q_{s}$.

The model is then applied to optical observations of the edge-on dust disk orbiting $\beta$ Pictoris, and good agreement is achieved when the unseen planetesimal disk is broad, with $75 \lesssim r \lesssim 150$ AU. If it is assumed that the dust grains are bright like Saturn's icy rings $\left(Q_{s}=0.7\right)$, then the cross section of dust in the disk is $A_{d} \simeq 2 \times 10^{20} \mathrm{~km}^{2}$ and its mass is $M_{d} \simeq 11$ lunar masses. In this case the planetesimal disk's dust production rate is quite heavy, $\dot{M}_{d} \sim 9 \mathrm{M}_{\oplus} / \mathrm{Myr}$, implying that there is or was a substantial amount of planetesimal mass there, at least 110 earth-masses. But if the dust grains are darker than assumed, then the planetesimal disk's mass-loss rate and its total mass are heavier. In fact, the apparent dearth of any major planets in this region, plus the planetesimal disk's heavy mass-loss rate, suggests that the $75 \lesssim r<150$ AU zone at $\beta$ Pic might be a region of planetesimal destruction, rather than a site of ongoing planet formation.
\end{abstract}

\title{
1. Introduction
}

A debris disk is a dusty circumstellar disk that is often found in orbit about a young star. It is also suspected that these dust disks might be sites of ongoing planet formation. This thinking is motivated by the dust grains' lifetime due to collisions, which is often much shorter than the age of the host star. Evidently, a circumstellar debris disk is also being supplied 
with fresh dust, and collisions among unseen planetesimals provides a plausible explanation for this dust production. And because planetesimals are also the seeds of planets, it is conceivable that a debris disk might be forming planets as well.

But keep in mind that if the collisional grinding in the planetesimal disk is too vigorous, then it is possible that the planetesimals might instead grind away before they have a chance to assemble into planets. Indeed, models of the early evolution of the outer Solar System propose that the early Kuiper Belt, which is a swarm of comets orbiting beyond Neptune, was initially composed of $M_{K B} \sim 30$ Earth masses 1 (Kenyon 2002), which would be enough to allow for the formation of two Neptune-class planets. But that model also shows that runaway accretion during the next $t \sim 500$ Myrs only managed to produce a handful of Plutosized bodies, while the bulk of the planetesimal mass there remained locked in the smaller planetesimals. Meanwhile, collisions among the smaller bodies steadily ground much of that mass to dust that is then blown out of the system by radiation pressure, which also stalls further growth. The mass-loss rate implied by this collisional grinding is $\dot{M}_{d} \sim t / M_{K B} \sim 10^{13}$ $\mathrm{gm} / \mathrm{sec}$ (Stern \& Colwell 1997; Kenyon 2002). Another outer Solar System scenario is the Nice model, which requires its primordial Kuiper Belt to persist until $t \sim 700$ Myrs since formation, which is when the giant planets suddenly adjust their orbits and trigger the Late Heavy Bombardment (Gomes et al. 2005; Levison et al. 2008). Although the dynamical history of the Nice model is rather different from other models, it should be noted that this and most other models of the outer Solar System's early evolution predict that the primordial Kuiper Belt had a quiescent period lasting several hundreds of millions years, during which the Belt would have lost tens of Earth-masses of material due to collisional grinding and blowout of dust due to radiation pressure (Stern \& Colwell 1997; Kenyon 2002).

An interesting question is whether the observed circumstellar debris disks have mass-loss rates comparable to that predicted for our early Solar System. The answer to that question will then provide some guidance as to whether these disks should instead be thought of potential sites for planet formation, or else regions of planetesimal destruction. To address this issue, the following develops a model that follows the time-evolution of a circumstellar debris disk. Sections 2.1 2.4 derives in some detail the model's physics, but readers not interested in those details can skip those sections. Sections 2.4.3 2.4.6 examines how the debris disk's structure and appearance depends upon the system parameters, and those results can also be quickly gleaned by inspecting the figures there. Section 3 then applies the model to observations of the debris disk orbiting $\beta$ Pictoris, with Section 4 providing a summary of the findings.

\footnotetext{
${ }^{1}$ The Kenyon (2002) model recommends 10 Earth masses in a 6 AU-wide annulus centered at $r=35$ AU, but this should be multiplied by $\sim 3$ to account for the Kuiper Belt's full radial width.
} 


\section{The debris disk model}

Radiation pressure is the dominate perturbation on small dust grains orbiting in a circumstellar debris disk (c.f., Strubbe \& Chiang 2006), and the review in Section 2.1 shows how the resulting dust orbits are simple functions of grain size. Dust collision rates are derived in Sections 2.2 2.3, and Section 2.4 shows how to use those rates to calculate the time evolution of the debris disk's dust abundance. The remaining subsections then illustrate how the simulated debris disk varies with the model parameters.

\section{1. dust orbit elements}

An orbiting dust grain is characterized by its size parameter $\beta$, which is the ratio of stellar radiation pressure to gravity. For a spherical grain of radius $R$, the size parameter is

$$
\beta=\frac{3 L_{\star} Q_{r p}}{16 \pi G M_{\star} c \rho R}=0.57 Q_{r p}\left(\frac{L_{\star}}{L_{\odot}}\right)\left(\frac{M_{\star}}{M_{\odot}}\right)^{-1}\left(\frac{\rho}{1 \mathrm{gm} / \mathrm{cm}^{3}}\right)^{-1}\left(\frac{R}{1 \mu \mathrm{m}}\right)^{-1}
$$

where $L_{\star}$ and $M_{\star}$ are the star's luminosity and mass, $G$ is the gravitational constant, $c$ the speed of light, $\rho$ is the grain's volume density, and $L_{\odot}$ and $M_{\odot}$ refer to solar values (Burns et al. 1979). The grain's radiation pressure efficiency is $Q_{r p}=Q_{a}+(1-g) Q_{s}$ (Burns et al. 1979), where $Q_{a}$ is the efficiency of the grain's absorption of starlight and $Q_{s}$ is the efficiency of light scattering by the grain. The scattering asymmetry parameter $g$ is

$$
g=\int \Phi(\phi) \cos \phi d \Omega
$$

where the integral runs over all solid angles, and the phase function $\Phi(\phi)$ gives the proportion of light that is scattered through scattering angle $\phi$, which is the angle between the directions followed by the incident and scattered light. The phase function is normalized such that

$\int \Phi(\phi) d \Omega=1$, so $|g| \leq 1$. Forward scattering dust grains have values of $g>0$, backscattering grains have $g<0$, and isotropic light scattering has $g=0$. The model developed here will be applied to the $\beta$ Pictoris debris disk, whose dust grains are substantially larger than the wavelength of the incident light. This is the geometric optics limit, and energy conservation in this limit requires $Q_{a}+Q_{s}=1$, so

$$
Q_{r p}=1-g Q_{s}
$$

Evidently, $Q_{r p} \simeq 1$ except when $\left|g Q_{s}\right|$ is not small, which can occur if the dust grains are efficient forward or back scatters. Equation (1) shows that radiation pressure is significant for grains of radii $R \sim \mathcal{O}(1) \mu \mathrm{m}$ when orbiting a solar-type star, and is unimportant for 
grains with $R \gg 1 \mu \mathrm{m}$. Since radiation pressure has the same inverse-square force law as stellar gravity, the effects of radiation pressure are easily accounted for by substituting $G M_{\star} \rightarrow(1-\beta) G M_{\star}$ into all equations for the dust grains' motion.

Dust is manufactured when planetesimals collide. Consider a dust grain that is generated by colliding planetesimals that reside in nearly circular orbits of radius $r$. These planetesimals have a specific energy $E_{p}=$ kinetic + potential energy that is

$$
E_{p}=\frac{1}{2} v^{2}-\frac{G M_{\star}}{r}=-\frac{G M_{\star}}{2 r}
$$

where $v=\sqrt{G M_{\star} / r}$ is the planetesimal's velocity, assuming its orbit is circular or nearly so. However, a dust grain that forms via a collision at radius $r$ will have a specific energy $E_{d}=\frac{1}{2} v^{2}-G M_{\star}(1-\beta) / r=-G M_{\star}(1-\beta) / 2 a$, where $a$ is the dust grain's semimajor axis. This assumes that the dust grain has the same velocity $v$ as its parent planetesimal at the moment of its creation, or equivalently, the velocity at which the grain is ejected from the planetesimal is small compared to its orbital speed. But this is reasonable since the debris disks considered here are thin, which implies that dust generation occurs with low ejection speeds. In this case, $\frac{1}{2} v^{2}=G M_{\star} / 2 r$ in the above, so the dust grain's semimajor axis is (Thébault et al. 2003)

$$
a(\beta)=\frac{1-\beta}{1-2 \beta} r .
$$

Bound orbits have $a>0$, so only dust having $\beta<\beta_{\max }$ where $\beta_{\max }=\frac{1}{2}$ will populate the resulting debris disk, while dust having $\beta \geq \beta_{\max }$ are not bound to the star and quickly leave the system. According to Equation (1) , the smallest bound grains have radii

$$
R_{\text {min }}=\frac{3 L_{\star} Q_{r p}}{16 \pi \beta_{\max } G M_{\star} c \rho}=1.1 Q_{r p}\left(\frac{L_{\star}}{L_{\odot}}\right)\left(\frac{M_{\star}}{M_{\odot}}\right)^{-1}\left(\frac{\rho}{1 \mathrm{gm} / \mathrm{cm}^{3}}\right)^{-1} \mu \mathrm{m} .
$$

Next, note that a planetesimal's specific angular momentum $L_{p}=r v$ also equals the dust grain's angular momentum $L_{d}$, since both have the same position and velocity at the moment of the grain's creation. Since a dust grain's Keplerian orbit has $L_{d}=\sqrt{G M_{\star}(1-\beta) a\left(1-e^{2}\right)}$ while the planetesimal's $L_{p}=\sqrt{G M_{\star} r}=L_{d}$, this provides the grain's eccentricity

$$
e(\beta)=\frac{\beta}{1-\beta}
$$

(Thébault et al. 2003). The dust grain's periapse $q_{\text {peri }}$ and apoapse $Q_{\text {apo }}$ distances are then

$$
\begin{aligned}
q_{\text {peri }} & =a(1-e)=r \\
\text { and } \quad Q_{\text {apo }} & =a(1+e)=r /(1-2 \beta)
\end{aligned}
$$


where $r$ is the orbital radii of the planetesimals that gave birth to the dust. Also note that periapse is precisely at the planetesimal's orbit, which tells us that the grain's longitude of periapse $\tilde{\omega}$ is also the longitude where the grain formed.

\section{2. $\quad$ streamlines}

Assessing the collisions that a dust grain experiences over its lifetime can be a laborious calculation, one that usually requires evaluating integrals over the dust grains' size distribution and the debris disk's three dimensional volume. However those integrals can be replaced by a simple summation when the model disk is quantized; see for example Krivov et al. (2006). In the following, the planetesimal disk, which is the source of this dust, is represented by $N_{r}$ concentric rings that have radii $r$ uniformly distributed over the interval $r_{\text {in }} \leq r \leq r_{\text {out }}$. Each planetesimal ring also produces dust at $N_{l}$ discreet sites that are distributed uniformly in longitude about the ring. The dust grains' radii $R=\left(\beta_{\max } / \beta\right) R_{\min }$ are also quantized so that they uniformly sample the interval $R_{\min }<R<R_{\max }$, with $N_{\beta}$ size-bins in the interval $0<\beta<\beta_{\max }$. As a result, the planetesimal disk produces dust whose grains inhabit $N_{s}=N_{r} N_{l} N_{\beta}$ distinct streamlines, or orbits, whose shapes are given by Equations (5) and (7), and whose orientations are uniformly distributed in longitude.

The rate of dust production $p(R, r)$ by the planetesimal disk is also a power law, with $p(R, r) \propto R^{-q}$ being the rate at which the planetesimals inject dust of radii $R$ into a single streamline, with $q$ being the power law for the grains' differential size distribution. The model will also allow for the dust production rate to vary with the source planetesimals' radial distance $r$ as $p(R, r) \propto\left(r / r_{\text {out }}\right)^{c}$. A planetesimal disk having $c<0$ can then be thought of as experiencing 'inside-out' erosion, since the inner portion of that disk experiences greater dust production than its outer parts, while a disk having $c>0$ suffers 'outside-in' erosion. Also note that $R \propto \beta^{-1}$, so the dust production rate can be written $p(\beta, r)=p_{0}\left(\beta / \beta_{\text {max }}\right)^{q}\left(r / r_{\text {out }}\right)^{c}$ where $p_{0}$ is a constant.

Since there are $N_{s}=N_{r} N_{l} N_{\beta}$ streamlines in the model debris disk, the index $i$ will be used to identify any one such dusty streamline, where $1 \leq i \leq N_{s}$. The streamlines' longitudes of periapse $\tilde{\omega}_{i}$ are uniformly distributed such that the resulting debris disk has $N_{l}$-fold symmetry. The quantity $n_{i}(t)$ will be the number of dust grains inhabiting streamline $i$ at time $t$; these grains all have the same size $\beta_{i}$ and orbit elements $a_{i}, e_{i}, \tilde{\omega}_{i}$, and they all formed at the same longitude $\tilde{\omega}_{i}$ in the same planetesimal ring that has radius $r_{i}$. The streamline's abundance $n_{i}(t)$ will then evolve over time $t$ due to the production of dust by the planetesimal ring $r_{i}$, but that quantity will also vary due to collisions with the dust grains that inhabit the disk's other streamlines. The rate at which these dust grains collide 


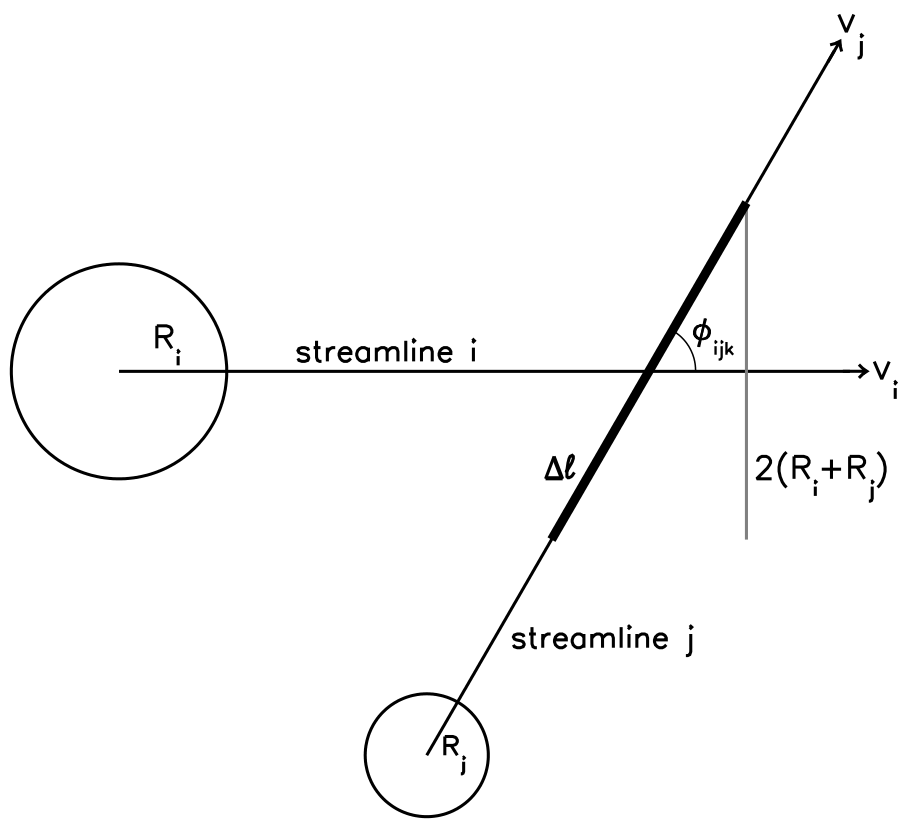

Fig. 1.- A dust grain of radius $R_{i}$ crosses streamline $j$ that contains dust of radii $R_{j}$. Grain $i$ sweeps a length $\Delta \ell=2\left(R_{i}+R_{j}\right) / \sin \phi_{i j k}$ along streamline $j$ where $\sin \phi_{i j k}$ is the angle between the grains' velocity vectors $\mathbf{v}_{i}$ and $\mathbf{v}_{j}$.

and destroy each other is derived below.

\section{3. collision rates}

Figure 1 shows a dust grain in streamline $i$ as it crosses streamline $j$. The dust in these streamlines have radii $R_{i}$ and $R_{j}$ and velocities $\mathbf{v}_{i}$ and $\mathbf{v}_{j}$. Suppose for now that the debris disk is flat, and that all dust grains have zero inclinations. In this case, grain $i$ would sweep across a length $\Delta \ell=2\left(R_{i}+R_{j}\right) / \sin \phi_{i j k}$ in streamline $j$, where $\phi_{i j k}$ is the angle between vectors $\mathbf{v}_{i}$ and $\mathbf{v}_{j}$, so $\sin \phi_{i j k}=\left|\mathbf{v}_{i} \times \mathbf{v}_{j}\right| / v_{i} v_{j}$. This quantity depends not just on the streamlines' orbit elements $a_{i}, e_{i}$ and $a_{j}, e_{j}$, but also on the streamlines' relative longitude of periapse $\tilde{\omega}_{k}=\tilde{\omega}_{j}-\tilde{\omega}_{i}$, which is why the $k$ subscript is also introduced. 
The linear density of dust grains in streamline $j$ is $\lambda_{j}$, so particle $i$ will collide with $\lambda_{j} \Delta \ell$ particles as it traverses streamline $j$. The flux of dust in this streamline is $\lambda_{j} v_{j}$, which is the rate at which grains cross a point in that streamline, so $\lambda_{j} v_{j}=n_{j} / T_{j}$ where $n_{j}$ is the total number of grains in that streamline and $T_{j}=2 \pi \sqrt{a_{j}^{3} /\left(1-\beta_{j}\right) G M_{\star}}$ is that streamline's orbit period. So the number of collisions that grain $i$ suffers as it crosses this site in streamline $j$ is $\Delta n_{i j k}=\lambda_{j} \Delta \ell=2\left(R_{i}+R_{j}\right) n_{j} f_{z} / \sin \phi_{i j k} v_{j} T_{j}$, where the factor $f_{z}$ is now introduced to account for the dust disk's vertical thickness. Note that $f_{z}$ would be unity if streamlines in the dust-disk where coplanar. However a real disk has a vertical half-width $h=I r_{i j k}$, where $I$ is the grains' characteristic inclination and $r_{i j k}$ is the radial distance from the star where streamlines $i$ and $j$ cross, with the $k$ subscript again indicating that that this quantity also depends on the streamlines' relative longitude of periapse $\tilde{\omega}_{k}$. Thus $f_{z}=2 f_{c}\left(R_{i}+R_{j}\right) / 2 h=f_{c}\left(R_{i}+R_{j}\right) / I r_{i j k}$ is the probability that two grains in streamlines $i$ and $j$ are close enough in the vertical direction to come in contact, and the factor $f_{c}=\pi / 4$ is a geometric correction that accounts for the grain's circular cross-section. And since there are $n_{i}$ grains in streamline $i$, they will suffer $n_{i} \Delta n_{i j k}$ collisions with streamline $j$ after one orbit period $T_{i}$, so the grains in streamline $i$ collide with the grains in streamline $j$ at site $r_{i j k}$ at the rate $n_{i} \Delta n_{i j k} / T_{i}$. Thus the total rate at which grains in streamline $i$ suffer collisions with all of the disk's other streamlines is

$$
\mathcal{R}_{i}=\sum \frac{n_{i} \Delta n_{i j k}}{T_{i}}=\frac{n_{i}}{T_{\text {out }}} \sum_{j=1}^{N_{r} N_{\beta}} \sum_{k=1}^{N_{l}} \sum_{r_{1}}^{r_{2}} \alpha_{i j k} n_{j},
$$

where the coefficient $\alpha_{i j k}=\pi\left(R_{i}+R_{j}\right)^{2} T_{\text {out }} f_{i j k}^{c} / 2 I \sin \phi_{i j k} r_{i j k} v_{j} T_{i} T_{j}$, and $T_{\text {out }}=2 \pi \sqrt{r_{\text {out }}^{3} / G M_{\star}}$ is the orbit period of the outermost planetesimal ring at $r=r_{\text {out }}$. Note that an additional factor $f_{i j k}^{c}$ was also introduced into the above; it takes values of $f_{i j k}^{c}=1$ if the collision with the impacting grain $R_{j}$ is energetic enough to disrupt the target grain $R_{i}$, and with $f_{i j k}^{c}=0$ if the collision does not disrupt grain $R_{i}$. The threshold for collisional disruption is given later in Section 2.3.2.

The leftmost sum in the above runs over streamlines that are composed of dust of radii $R_{j}$ that have size parameters $\beta_{j}=\beta_{\max }\left(R_{\min } / R_{j}\right)$ produced by the planetesimal ring that has radius $r_{j}$; their orbit elements $a_{j}$ and $e_{j}$ are given by Equations (5) and (7). These dust grains have $N_{\beta}$ possible sizes, and there are $N_{r}$ such planetesimal rings, so $1 \leq j \leq N_{\beta} N_{r}$. The middle sum then accounts for the $N_{l}$ sites in each planetesimal ring that produce dust having relative longitudes $\tilde{\omega}_{k}=\tilde{\omega}_{j}-\tilde{\omega}_{i}$, so $1 \leq k \leq N_{l}$. Also keep in mind that pairs of orbits cross at two sites $r_{i j k}=r_{1}$ and $r_{i j k}=r_{2}$ whose contributions are accounted for by the rightmost sum.

But first a comment on differently-sized dust grains that are produced at the same site within the same planetesimal ring. Such grains have the same longitude of periapse, $\tilde{\omega}_{i}=\tilde{\omega}_{j}$, 
and thus will re-encounter each other at periapse with the same velocity $\mathbf{v}_{i}=\mathbf{v}_{j}$. This also

makes $\sin \phi_{i j k}=0$, which might appear problematic because the collision probability $\alpha_{i j k}$ would seem to be singular there. But keep in mind that grains $i$ and $j$ have the same velocity at periapse, namely, the planetesimal ring's velocity. Consequently, these grains have zero relative velocity at this particular site, so there is no chance for collisional fragmentation, and $\alpha_{i j k}$ is set to zero in this instance.

A dust grain's velocity is $v_{j}=\left(2 \pi r_{\text {out }} / T_{\text {out }}\right) \sqrt{\left(1-\beta_{j}\right)\left(2 r_{\text {out }} / r_{i j k}-r_{\text {out }} / a_{j}\right)}$ where $r_{i j k}$ is the distance from the star where streamlines $i$ and $j$ cross. Since $T_{i} / T_{\text {out }}=\left(a_{i} / r_{\text {out }}\right)^{3 / 2} / \sqrt{1-\beta_{i}}$, the $\alpha_{i j k}$ in the above then becomes

$$
\alpha_{i j k}=\frac{\beta_{\max }^{2} f_{i j k}^{c}}{4 I \sin \phi_{i j k}} \sqrt{\frac{1-\beta_{i}}{2 a_{j} / r_{i j k}-1}}\left(\frac{\beta_{i}+\beta_{j}}{\beta_{i} \beta_{j}}\right)^{2}\left(\frac{r_{\text {out }}^{2}}{r_{i j k} a_{j}}\right)\left(\frac{r_{\text {out }}}{a_{i}}\right)^{3 / 2}\left(\frac{R_{\text {min }}}{r_{\text {out }}}\right)^{2} .
$$

This quantity is the collision probability density, in the sense that $\alpha_{i j k} n_{j}$ is the probability per time $T_{\text {out }}$ that a dust grain in streamline $i$ suffers a collision with the dust in streamline $j$ at site $r_{i j k}$.

\subsection{1. orbit crossing sites}

The $r_{i j k}$ in Equation (10) is one of two sites where orbit $i$ crosses orbit $j$, and it is sensitive to the orbits' relative longitude of periapse $\tilde{\omega}_{k}=\tilde{\omega}_{j}-\tilde{\omega}_{i}$. To solve for $r_{i j k}$, rotate the coordinate system so that the $\hat{\mathbf{x}}$ axis points to orbit $i$ 's periapse. Requiring the two orbital ellipses to intersect yields

$$
r_{i j k}=\frac{p_{i}}{1+e_{i} \cos \theta}=\frac{p_{j}}{1+e_{j} \cos \left(\theta-\tilde{\omega}_{k}\right)}
$$

where $p_{i}=a_{i}\left(1-e_{i}\right)$ is the orbit's semilatus rectum and $\theta$ is measured from the $\hat{\mathbf{x}}$ direction. Using $\cos \left(\theta-\tilde{\omega}_{k}\right)=\cos \theta \cos \tilde{\omega}_{k}+\sin \theta \sin \tilde{\omega}_{k}$ allows Equation (11) to be written as $A \cos \theta-$ $B \sin \theta+C=0$, where $A=e_{i}-p e_{j} \cos \tilde{\omega}_{k}, B=p e_{j} \sin \tilde{\omega}_{k}, C=1-p$, and $p=p_{i} / p_{j}$. Solving for $\sin \theta^{2}=1-\cos \theta^{2}$ then yields $\cos \theta^{2}+2 D \cos \theta+E=0$ where $D=A C /\left(A^{2}+B^{2}\right)$ and $E=\left(C^{2}-B^{2}\right) /\left(A^{2}+B^{2}\right)$, which has solution

$$
\begin{aligned}
\cos \theta & =-D \pm \sqrt{D^{2}-E} \\
\text { and } \sin \theta & =(A \cos \theta+C) / B
\end{aligned}
$$

Solve Equations (12) for the two $\theta$ 's and insert those into $r_{i j k}=p_{i} /\left(1+e_{i} \cos \theta\right)$ to get the radial distances for where these orbits cross. Then rotate the coordinate system back, $\theta \rightarrow \theta+\tilde{\omega}_{i}$, to get the longitudes where these orbits cross. Note that Equations (12) are real when $D^{2} \geq E$, which is the requirement for orbits $i$ and $j$ to cross. 
To evaluate the $\sin \phi_{i j k}$ in Equation (10), the dust grain's radial velocity $v_{i, r}$ and tangential velocity $v_{i, \theta}$ need to be evaluated at the collision site. Those velocity components are

$$
\begin{aligned}
v_{i, r} & =\frac{e_{i} a_{i} \Omega_{i}}{\sqrt{1-e_{i}^{2}}} \sin \left(\theta-\tilde{\omega}_{i}\right) \\
\text { and } \quad v_{i, \theta} & =\frac{a_{i} \Omega_{i}}{\sqrt{1-e_{i}^{2}}}\left[1+e_{i} \cos \left(\theta-\tilde{\omega}_{i}\right)\right]
\end{aligned}
$$

where $\Omega_{i}=2 \pi / T_{i}$ is the grains' mean motion. These velocity components are then used to obtain $\sin \phi_{i j k}=\left|\mathbf{v}_{i} \times \mathbf{v}_{j}\right| / v_{i} v_{j}=\left|v_{i, r} v_{j, \theta}-v_{i, \theta} v_{j, r}\right| / v_{i} v_{j}$ where $v_{i}$ is the grain's total velocity.

Note that the above ignored the grain's small vertical velocity $v_{i, z}$, which is of order $I v_{i}$. This is appropriate since $I \ll 1$. However the following Section will still need an estimate of the dust grains' relative velocity in the vertical direction when determining whether collisions are destructive. And since the model being developed here does not faithfully follow the dust grains' vertical motions, it is assumed here that any two pairs of dust grains have a relative vertical speed that is equal to their rms value, $\left|v_{i, z}-v_{j, z}\right|=\sqrt{\left(I v_{i}\right)^{2}+\left(I v_{j}\right)^{2}}$.

\subsection{2. disruption threshold}

Now derive the threshold for a dust grain's collisional disruption. The target dust grain has mass $m_{i}$ and velocity $\mathbf{v}_{i}$, and it is struck by an impacting dust grain of mass $m_{j}$ and velocity $\mathbf{v}_{j}$. The two dust grains' relative velocity just prior to impact is $\mathbf{v}_{r}=\mathbf{v}_{j}-\mathbf{v}_{i}$, and the specific work that the impactor must do on the target grain in order to disrupt it is $Q^{\star}$. For simplicity, assume that any debris produced by the disruption of the target grain has zero dispersion velocity, which then provides the minimum energy that is needed for disruption. In the target dust grain's rest frame, the system's kinetic energy is $\frac{1}{2} m_{j} v_{r}^{2}$ just prior to impact. It is shown below that most collisions will shatter both the target and the impactor, so the post-impact debris will have a total energy $E=\frac{1}{2} m_{j} v_{r}^{2}-Q^{\star}\left(m_{i}+m_{j}\right)$ where $Q^{\star} m_{i}$ is the work that must do in order to shatter grain $m_{i}$. But this energy can also be written as $E=\frac{1}{2} m_{i} v_{i}^{\prime 2}+\frac{1}{2} m_{j} v_{j}^{\prime 2}$ where $v_{i}^{\prime}$ is the post-impact speed of the debris from grain $m_{i}$, and $v_{j}^{\prime}$ is the post-impact speed of mass $m_{j}$ in this reference frame. Momentum conservation also requires that $m_{j} v_{r}=m_{i} v_{i}^{\prime}+m_{j} v_{j}^{\prime}$. Solving the energy and momentum equations simultaneously then provides the post-impact speed of debris $m_{i}$,

$$
v_{i}^{\prime}=\frac{m_{j} v_{r}}{m_{i}+m_{j}}\left[1+\sqrt{1-\frac{2\left(m_{i}+m_{j}\right)^{2} Q^{\star}}{m_{i} m_{j} v_{r}^{2}}}\right] .
$$


Keep in mind that this is the speed of debris in the reference frame where mass $m_{i}$ was stationary just before impact. Consequently, the speed of that debris in the inertial reference frame is

$$
\mathbf{v}_{i}+v_{i}^{\prime} \hat{\mathbf{v}}_{r}
$$

where $\hat{\mathbf{v}}_{r}$ is the unit vector that points in the direction of $\mathbf{v}_{r}$.

The collision is energetic enough to disrupt grain $m_{i}$ when Equation (14) is real, which requires $v_{r}^{2} \geq 2\left(m_{i}+m_{j}\right)^{2} Q^{\star} / m_{i} m_{j}$. Assuming all grains are spheres of similar density, this requirement becomes

$$
v_{r}^{2}>\frac{2 Q^{\star}\left(\beta_{i}^{3}+\beta_{j}^{3}\right)^{2}}{\left(\beta_{i} \beta_{j}\right)^{3}}
$$

When the above is satisfied, the collision is fast enough to destroy the target grain $\beta_{i}$, which is accounted for in Equation (10) by setting $f_{i j k}^{c}=1$, while $f_{i j k}^{c}=0$ when the above is not satisfied. In that case grain $m_{i}$ will recoil from the collision and enter a new orbit about the star. However, nearly all collisions result in the disruption of grain $m_{i}$, so it is safe to ignore these rare non-disruptive collision events.

Unfortunately, the dust grains' collisional specific energy $Q^{\star}$ is rather uncertain, and likely depends on the target grain's size $R_{i}$ (Holsapple et al. 2002). Another difficulty is that experimental measurements of $Q^{\star}$ only extend down to cm-sized targets (Holsapple 1994). However models of observed debris disks suggest $Q^{\star} \sim 3 \times 10^{6} \mathrm{ergs} / \mathrm{gm}$ (Wyatt et al. 2007b), while Strubbe \& Chiang (2006) recommend a nominal value of $Q^{\star} \sim 10^{7} \mathrm{ergs} / \mathrm{gm}$ if the dust grains are rocky. But if circumstellar dust grains are icy, then values $\sim 100$ smaller are possible (Holsapple et al. 2002). Strubbe \& Chiang (2006) also note that sandblasting machines accelerate destructive particles to $v \sim 100 \mathrm{~m} / \mathrm{sec}$, which would suggest an upper limit of $Q^{\star} \sim v^{2} \lesssim 10^{8} \mathrm{erg} / \mathrm{gm}$. However, all collisions in this model are disruptive when $Q^{\star}<10^{6} \mathrm{ergs} / \mathrm{gm}$, so the following will only consider the interval $10^{6}<Q^{\star}<10^{8} \mathrm{ergs} / \mathrm{gm}$. And in the example model of Section 2.4.3, 99.7\% of all collisions are destructive when $Q^{\star}=10^{7} \mathrm{ergs} / \mathrm{gm}$ while $89 \%$ of collisions are destructive when $Q^{\star}=10^{8} \mathrm{ergs} / \mathrm{gm}$.

\section{4. the debris disk's time evolution}

The debris disk's dust abundance evolves due to dust production by the planetesimal rings minus losses due to collisions among dust grains. To quantify this, let $p_{i}=p\left(\beta_{i}, r_{i}\right)$ be the rate that one site in planetesimal ring $r_{i}$ produces dust of size $\beta_{i}$ that gets injected into streamline $i$. The abundance $n_{i}$ of dust in streamline $i$ then evolves according to the rate 
equation

$$
\frac{d n_{i}}{d t}=p_{i}-\mathcal{R}_{i}=p_{i}-\frac{n_{i}}{T_{\text {out }}} \sum_{j=1}^{N_{r \beta}} \sum_{k=1}^{N_{l}} \sum_{r_{1}}^{r_{2}} \alpha_{i j k} n_{j},
$$

where the left term accounts for dust production and the right term accounts for collisional destruction, Equation (9). Index $i$ refers to the dust that reside in the target streamline $i$, while $j$ refers to dust grains in the impacting streamline $j$ that have a relative longitude of periapse $\tilde{\omega}_{k}=\tilde{\omega}_{j}-\tilde{\omega}_{i}$. Although the model consists of $N_{s}=N_{r} N_{l} N_{\beta}$ streamlines, there are only $N_{r \beta}=N_{r} N_{\beta}$ equations in Equation (19) that are distinct, since the $N_{l}$ streamlines that are generated in the same planetesimal ring $r_{i}$ with the same dust size $\beta_{i}$ have an identical evolution due to the system's azimuthal symmetry.

The total number of dust grains of size $\beta_{i}$ that are generated by planetesimal ring $r_{i}$ is $N_{i}=N_{l} n_{i}$, where $N_{l}$ is the number of dust-producing sites in a planetesimal ring. Similarly, the total rate at which ring $r_{i}$ produces dust of size $\beta_{i}$ is $P_{i}=N_{l} p_{i}$. Multiplying Equation (17) by $N_{l}$ and setting

$$
\bar{\alpha}_{i j}=\frac{1}{N_{l}} \sum_{k=1}^{N_{l}} \sum_{r_{1}}^{r_{2}} \alpha_{i j k}
$$

then provides the rate at which $N_{i}$ evolves over time,

$$
\frac{d N_{i}}{d t}=P_{i}-\frac{N_{i}}{T_{\text {out }}} \sum_{j=1}^{N_{r \beta}} \bar{\alpha}_{i j} N_{j}
$$

Here, $\bar{\alpha}_{i j}$ is the mean probability per time $T_{\text {out }}$ that a grain of size $\beta_{i}$ and orbit elements $a_{i}, e_{i}$ collides with a grain of size and orbit $\beta_{j}, a_{j}, e_{j}$. Note that this is a mean probability since Equation (18) averages probabilities over all possible longitudes of periapse $\tilde{\omega}_{k}=\tilde{\omega}_{j}-\tilde{\omega}_{i}$.

This model is also going to assume that, when dust grains collide, the resulting dust fragments are so small that they are unbound and driven away by radiation pressure. This assumption simplifies the problem enormously since, if it where not true, then the number of streamlines in the debris disk would grow exponentially as collisions beget additional dusty streamlines that collide with other dust and generate even more streamlines. The validity of this assumption is confirmed later in Section 3.3.1.

\subsection{1. two-component model}

Now consider a rather simple system, that of a single planetesimal ring that manufactures dust having only two sizes, small (S) grains having radii near the blow-out radius $R_{\min }$, 
and large (L) grains of radii $R_{L} \gg R_{\text {min }}$. In the resulting debris disk, nearly all collisions are with the small grains, due to their much greater abundance in the disk, $N_{S} \gg N_{L}$, and their much greater production rates $P_{S} \gg P_{L}$. Because small grains are only colliding with other small grains, their abundance varies as

$$
\frac{d N_{S}}{d t}=P_{S}-\frac{\bar{\alpha}_{S S} N_{S}^{2}}{T_{\text {out }}}
$$

according to Equation (19), where $\bar{\alpha}_{S S}$ is the probability density for collisions among small grains. The solution is

$$
N_{S}(t)=N_{S}^{e q} \tanh \left(t / t_{S}\right)
$$

where

$$
N_{S}^{e q}=\sqrt{\frac{P_{S} T_{\text {out }}}{\bar{\alpha}_{S S}}} \quad \text { and } \quad t_{S}=\sqrt{\frac{T_{\text {out }}}{\bar{\alpha}_{S S} P_{S}}}=\frac{N_{S}^{e q}}{P_{S}} .
$$

Here, $N_{S}^{e q}$ is the total number of small dust grains that result when the disk settles into equilibrium, which occurs at time $t \gg t_{S}$ when $\tanh \left(t / t_{S}\right) \rightarrow 1$ and $d N_{S} / d t \rightarrow 0$. Note that $t_{S}$ is also the small dust grains' collisional lifetime. Evidently the small grains' equilibrium abundance as well as their optical depth will vary as $N_{S}^{e q} \propto P_{S}^{1 / 2}$ where $P_{S}$ is their production rate, while the timescale for the disk to settle into collisional equilibrium varies as $t_{S} \propto P_{S}^{-1 / 2}$.

The large grains' abundance $N_{L}$ also evolves according to Equation (19), which becomes $d N_{L} / d t=P_{L}-\bar{\alpha}_{L S} N_{L} N_{S} / T_{\text {out }}$ when collisions with small grains dominate. And when the disk has settled into collisional equilibrium, $d N_{L} / d t=0$, so the equilibrium abundance of large grains is

$$
N_{L}^{e q}=\frac{P_{L} T_{o u t}}{\bar{\alpha}_{L S} N_{S}^{e q}}
$$

where $\bar{\alpha}_{L S}$ is the large grain's collisional probability density.

Although this two-component treatment might seem too simple for quantitative work, it is illustrative, because a real disk's optical depth and collision rates are often dominated by the disk's smallest grains that have size parameters close to $\beta_{\max }$. In that case, the disk's collisional equilibrium timescale and its optical depth would all vary with the square root of the planetesimal's dust production rate. Equations (22 23) are also useful because they provide a convenient test of the more general debris disk model that is developed below in Section 2.4.2, using that more general model to simulate a two-component debris yields results that agree with Equations (21 23) to within $0.04 \%$. 


\subsection{2. scale-invariant evolution}

Now consider a more realistic scenario where the system's planetesimal rings produce dust whose rates are power-laws in the dust size parameter $\beta_{i}$ and planetesimal ring radius $r_{i}$ such that $P_{i}=P_{0}\left(\beta_{i} / \beta_{\max }\right)^{q}\left(r_{i} / r_{\text {out }}\right)^{c}$. It will also be convenient to first convert Equation (19) into a dimensionless system of equations via the substitutions

$$
t=T_{0} t^{\star} \quad \text { and } \quad N_{i}(t)=N_{0}\left(\frac{\beta_{i}}{\beta_{\max }}\right)^{q}\left(\frac{r_{i}}{r_{\text {out }}}\right)^{c} N_{i}^{\star}\left(t^{\star}\right)
$$

where $t^{\star}=t / T_{0}$ is dimensionless time coordinate and $N_{i}^{\star}\left(t^{\star}\right)=N_{i}(t) P_{0} / P_{i} N_{0}$ is the scaled abundance of grains of size $\beta_{i}$ produced by ring $r_{i}$. The collision probability density will also be written as $\bar{\alpha}_{i j}=\left(\bar{\alpha}_{i j}^{\star} / I\right)\left(\beta_{j} / \beta_{\text {max }}\right)^{-q}\left(r_{j} / r_{\text {out }}\right)^{-c}\left(R_{\text {min }} / r_{\text {out }}\right)^{2}$ where the scaled collision probability is

$$
\bar{\alpha}_{i j}^{\star}=\frac{1}{N_{l}} \sum_{k=1}^{N_{l}} \sum_{r_{1}}^{r_{2}} \frac{\beta_{\text {max }}^{2} f_{i j k}^{c}}{4 \sin \phi_{i j k}} \sqrt{\frac{1-\beta_{i}}{2 a_{j} / r_{i j k}-1}}\left(\frac{\beta_{i}+\beta_{j}}{\beta_{i} \beta_{j}}\right)^{2}\left(\frac{\beta_{j}}{\beta_{\text {max }}}\right)^{q}\left(\frac{r_{\text {out }}^{2}}{r_{i j k} a_{j}}\right)\left(\frac{r_{\text {out }}}{a_{i}}\right)^{3 / 2}\left(\frac{r_{j}}{r_{\text {out }}}\right)^{c} .
$$

In the above, the constant timescale $T_{0}$ and population scale-factor $N_{0}$ are

$$
\begin{aligned}
T_{0} & =\sqrt{\frac{I T_{\text {out }}}{P_{0}}}\left(\frac{r_{\text {out }}}{R_{\text {min }}}\right) \\
\text { and } N_{0} & =P_{0} T_{0}=\sqrt{I P_{0} T_{\text {out }}}\left(\frac{r_{\text {out }}}{R_{\text {min }}}\right) .
\end{aligned}
$$

Inserting this into Equation (19) then yields a system of equations

$$
\frac{d N_{i}^{\star}}{d t^{\star}}=1-N_{i}^{\star} \sum_{j=1}^{N_{r \beta}} \bar{\alpha}_{i j}^{\star} N_{j}^{\star}
$$

that is not just dimensionless, but also scale invariant. This is very handy, since Equation (27) need only be solved once in order to apply the result to a variety of systems that might have a range of planetesimal radii $r_{\text {out }}$ orbiting stars of varied masses $M_{\star}$ and luminosities $L_{\star}$. Note, however, that the rescaled collision probability $\bar{\alpha}_{i j}^{\star}$ does depend of the dust power laws $q$ and $c$, the dust grains' specific energy for collisional disruption $Q^{\star}$, and the planetesimal disk's radial width $r_{i n} / r_{\text {out }}$ (Equation 25), so any changes to those parameters does require solving Equation (27) again.

Equation (27) is a coupled set of nonlinear differential equations whose initial conditions are $N_{i}^{\star}(0)=0$. These equations are easily solved numerically for each streamline's relative abundance $N_{i}^{\star}\left(t^{\star}\right)$ using a Runga-Kutta algorithm. That solution to Equation (27) then 
provides the dust grains' relative abundances $N_{i}(t) / N_{0}=\left(P_{i} / P_{0}\right) N_{i}^{\star}\left(t^{\star}\right)$. A sample calculation is shown in Figure 2, which plots the relative abundances $N_{i} / N_{0}$ versus dimensionless time $t^{\star}$ for dust that is generated by a narrow planetesimal ring $\left(N_{r}=1\right.$ and $\left.r_{\text {in }}=r_{\text {out }}\right)$ whose dust production has the $q=3.5$ size distribution that expected for debris that is in collisional equilibrium (Dohnanyi 1969). In this and all calculations that follow, the simulated debris disk is composed of grains having $N_{\beta}=200$ distinct sizes, with the dust size parameters distributed over the interval $0.0652 \leq \beta_{i} \leq 0.497$ such that the dust grains' radii $R_{i}=\left(\beta_{\max } / \beta_{i}\right) R_{\min }$ are uniformly sampled over the interval $R_{\min } \leq R \leq R_{\max }$. Dust of each size $\beta_{i}$ are launched from $N_{l}=100$ evenly-spaced longitudes about the planetesimal disk, so the resulting debris disk is comprised of $N_{s}=N_{r} N_{l} N_{\beta}=2 \times 10^{4} N_{r}$ distinct streamlines. The planetesimal disk that is the source of this dust is usually composed of $N_{r}=3$ to 5 planetesimal rings, except $N_{r}=1$ is used when the dust source is a narrow planetesimal ring, as is the case for Figure 2, The $\beta$ distribution used here results in streamlines that range as far as $r=150 r_{\text {out }}$ from the central star, where $r_{\text {out }}$ is the planetesimal disk's outer radius. Execution times on a desktop PC range from 4 minutes for simulations having $N_{r}=1$ to 2 hours when $N_{r}=5$. 


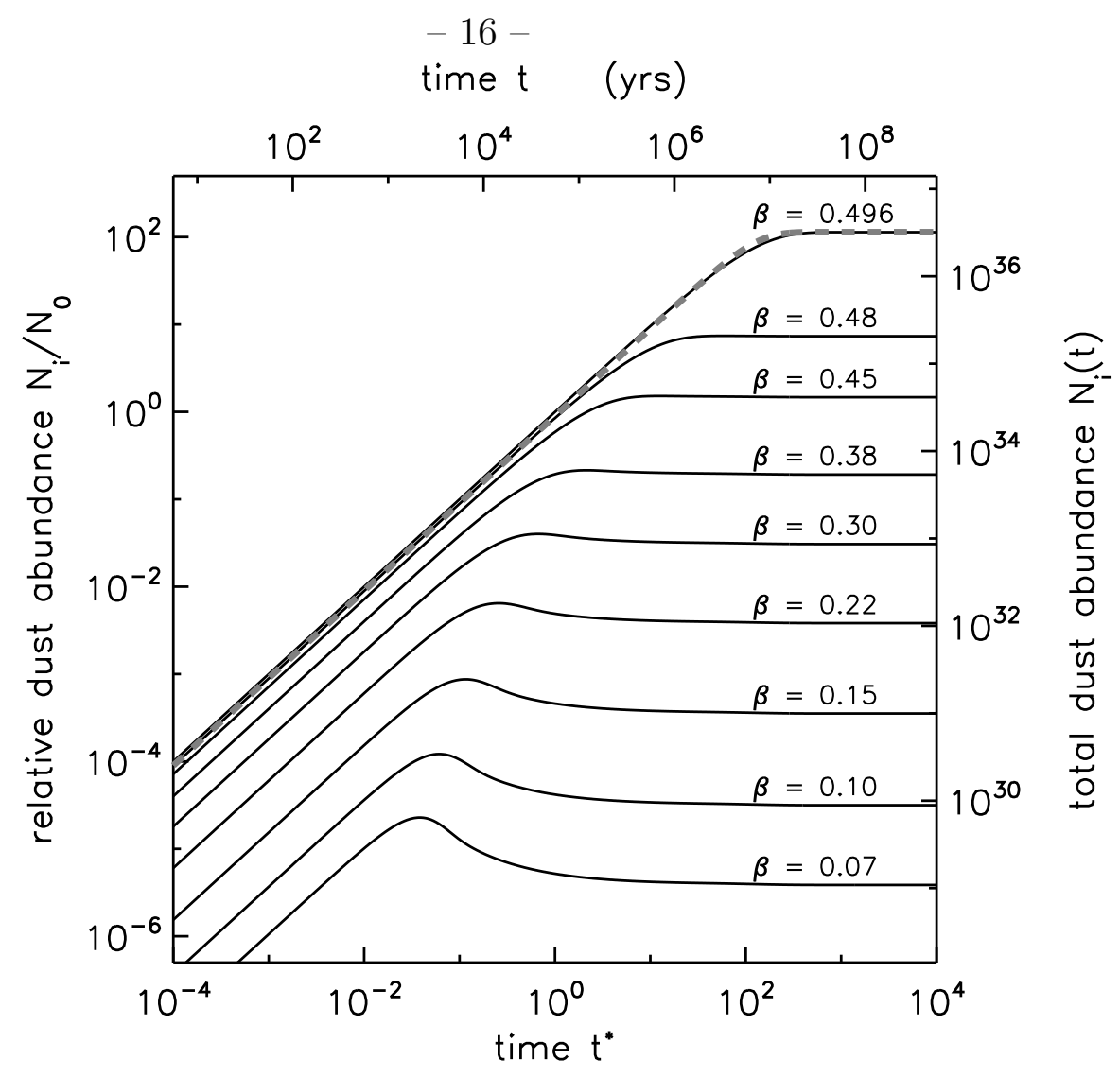

Fig. 2.- Equation (27) is solved for the scaled dust abundances $N_{i}^{\star}\left(t^{\star}\right)$ assuming that the dust is produced by a narrow planetesimal ring $\left(N_{r}=1\right)$ with a $q=3.5$ size distribution. The scaled dust abundances are then converted into absolute dust abundances $N_{i}\left(t^{\star}\right) / N_{0}$ and plotted versus dimensionless time $t^{\star}$ (left and lower axes). This model also assumed that the dust are weak, $Q^{\star}=10^{6} \mathrm{ergs} / \mathrm{gm}$, so all collisions disrupt the dust. Abundances for nine selected streamlines having the indicated size parameter $\beta$ are shown above; not shown are the model's 191 other curves that behave similarly. The right and upper axes are scaled for an $r_{\text {out }}=50 \mathrm{AU}$ planetesimal ring in orbit about a solar-type star that produces dust at the rate $\dot{M}_{d}=10^{13} \mathrm{gm} / \mathrm{sec}$ with $\rho=1 \mathrm{gm} / \mathrm{cm}^{3}, Q_{r p}=0.96$, and $I=0.1$ radians, for which $N_{0}=2.8 \times 10^{34}$ grains and $T_{0}=5.6 \times 10^{4} \mathrm{yrs}$. The dashed grey curve is Equation (21) with an equilibrium timescale $T_{e q}=7.1$ Myrs.

Although the calculation seen in Figure 2 covers a fairly broad range of $\beta$ parameters, the resulting debris disk is nonetheless populated by dust having a fairly narrow range of sizes, since it is only the smallish dust grains that are lofted well outside of the birth ring by radiation pressure. In Figure 2, the central star is solar and the dust grains have a density $\rho=1 \mathrm{gm} / \mathrm{cm}^{3}$ and radiation pressure efficiency $Q_{r p}=0.96$, so the radius of the smallest bound dust grain is $R_{\min }=1.1 \mu \mathrm{m}$, and the range of dust radii that populate the simulated debris disk is only $1.1 \mu \mathrm{m} \leq R \leq 8.5 \mu \mathrm{m}$. Of course, a real disk will also manufacture larger dust, but those larger grains would be confined to the disk's innermost 
region $r_{\text {in }} \leq r \leq 1.15 r_{\text {out }}$, so they do not alter the disk's large-scale structure at $r \gg r_{\text {out }}$. Also, these larger grains contribute little to the disk's optical depth due to their slower dust production rates and their very short collisional lifetime, so their absence from these simulations is justified.

The parameter $P_{0}$ that appears in the constants $N_{0}$ and $T_{0}$ (Equations 26) is related to the planetesimal disk's total dust mass production rate, which is $\dot{M}_{d}=\sum_{i} P_{i} \frac{4 \pi}{3} \rho R_{i}^{3}$ where the sum proceeds over all streamlines that make up the dust disk. Since $P_{i}=$ $P_{0}\left(\beta_{i} / \beta_{\text {max }}\right)^{q}\left(r_{i} / r_{\text {out }}\right)^{c}$, this provides $P_{0}=\dot{M}_{d} / S_{q c} m_{\text {min }}$, where $m_{\min }=\frac{4 \pi}{3} \rho R_{\text {min }}^{3}$ is the mass of the smallest bound dust grain, and the factor $S_{q c}=\sum_{i}\left(\beta_{i} / \beta_{\text {max }}\right)^{q-3}\left(r_{i} / r_{\text {out }}\right)^{c}$. Inserting this and Equation (66) into (26) then provides the scale factors

$$
\begin{aligned}
N_{0}= & \frac{2.8 \times 10^{34}}{Q_{r p}^{5 / 2}}\left(\frac{\dot{M}_{d}}{10^{13} \mathrm{gm} / \mathrm{sec}}\right)^{1 / 2}\left(\frac{I}{0.1 \mathrm{rad}}\right)^{1 / 2}\left(\frac{L_{\star}}{L_{\odot}}\right)^{-5 / 2}\left(\frac{M_{\star}}{M_{\odot}}\right)^{9 / 4} \\
& \times\left(\frac{\rho}{1 \mathrm{gm} / \mathrm{cm}^{3}}\right)^{2}\left(\frac{r_{\text {out }}}{50 \mathrm{AU}}\right)^{7 / 4}\left(\frac{S_{q c}}{107}\right)^{-1 / 2} \\
\text { and } T_{0}= & 5.6 \times 10^{4} Q_{r p}^{1 / 2}\left(\frac{\dot{M}_{d}}{10^{13} \mathrm{gm} / \mathrm{sec}}\right)^{-1 / 2}\left(\frac{I}{0.1 \mathrm{rad}}\right)^{1 / 2}\left(\frac{L_{\star}}{L_{\odot}}\right)^{1 / 2}\left(\frac{M_{\star}}{M_{\odot}}\right)^{-3 / 4} \\
& \times\left(\frac{r_{\text {out }}}{50 \mathrm{AU}}\right)^{7 / 4}\left(\frac{S_{q c}}{107}\right)^{1 / 2} \mathrm{yrs}
\end{aligned}
$$

where the factor $S_{q c}=107,320$, and 530 for the simulations having $N_{r}=1,3$, and 5 planetesimal rings with $c=0$ and $q=3.5$. The above quantities allow one to easily rescale all figures shown here for systems having alternate dust production rate $\dot{M}_{d}$ or the planetesimal disk's outer radius $r_{\text {out }}$, etc. The following subsection will also show that the time $T_{e q}$ for the resulting dust-disk to settle into an equilibrium where dust production balances mass-loss due to collisions is $T_{e q} \simeq 130 T_{0}$.

\subsection{3. example: $a_{r}=50$ AU birth ring}

To illustrate, consider a narrow $\left(N_{r}=1\right)$ planetesimal ring of radius $r_{\text {out }}=50 \mathrm{AU}$ in orbit about a solar-type star. This ring will have a dust mass production rate of $\dot{M}_{d}=10^{13}$ $\mathrm{gm} / \mathrm{sec}$, with dust having a density $\rho=1 \mathrm{gm} / \mathrm{cm}^{3}$ and inclinations $I=0.1$ radians. The grains are dark, with $Q_{s}=0.1$, and are asymmetric light scatters having $g=0.4$, so the radiation pressure efficiency is $Q_{r p}=0.96, N_{0}=2.8 \times 10^{34}$ and $T_{0}=5.6 \times 10^{4}$ yrs. Multiplying the lower and left axes in Figure 2 by these constants then provides the dust abundances $N_{i}(t)$ versus physical time $t$, which can also be read off the right and upper axes of Figure 2. That figure shows that the smallest dust grains having $\beta \simeq \beta_{\max }$ need the most time 


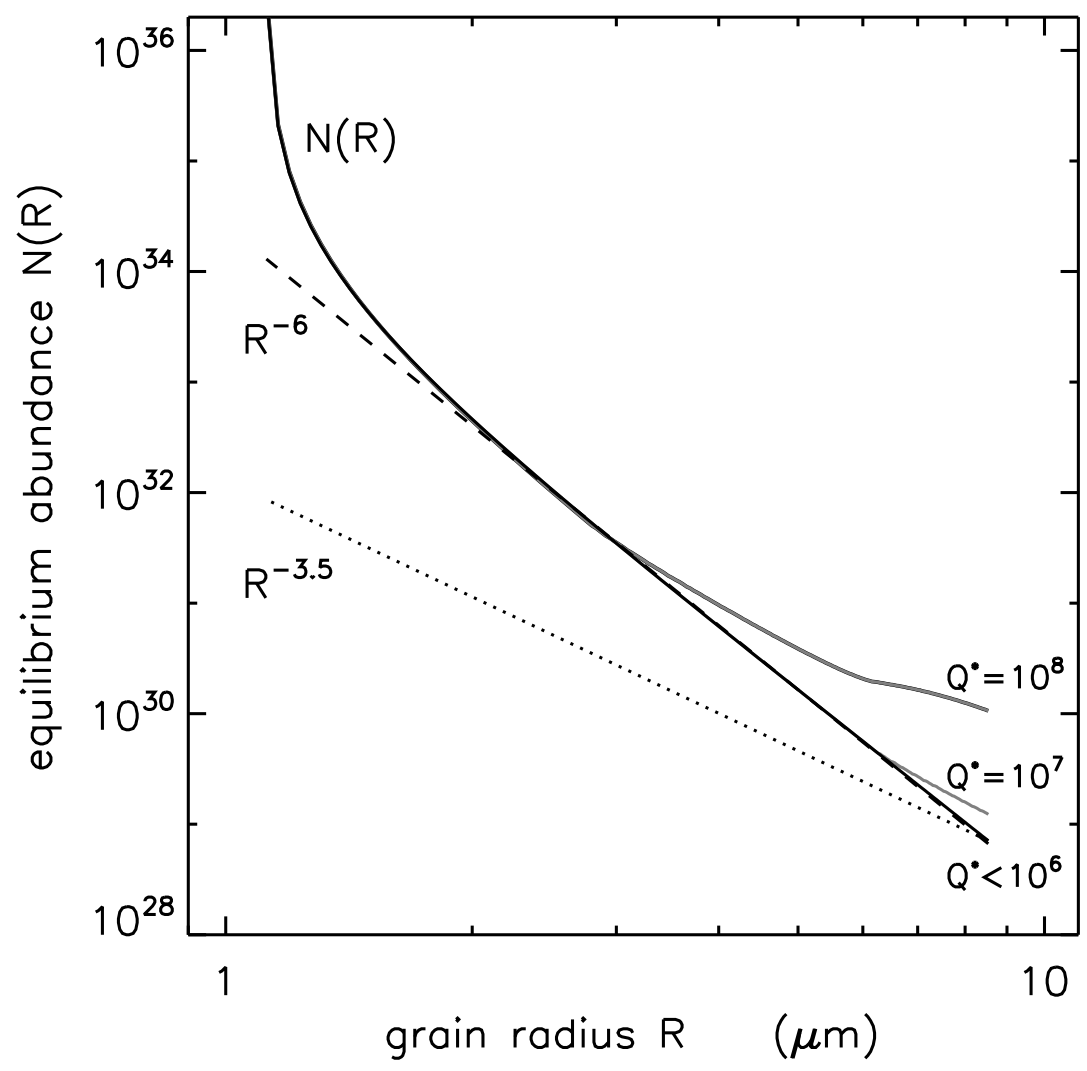

Fig. 3.- The black $N(R)$ curve is the differential size distribution for the dust in the debrisdisk model of Figure 2 after the disk has achieved equilibrium at times $t \gg T_{e q}$ assuming the dust grains are weak with $Q^{\star}<10^{6} \mathrm{ergs} / \mathrm{gm}$. Grey curves are for models having stronger dust grains, $Q^{\star}=10^{7}$ and $10^{8} \mathrm{ergs} / \mathrm{gm}$. The dashes indicates an $R^{-6}$ size distribution, and the dotted curve is proportional to the planetesimal ring's differential dust production rate $P(R) \propto R^{-3.5}$.

to settle into an equilibrium where dust production balances destruction due to collisions. The grey dashed curve in Figure 2 also shows that the abundance of the smallest dust does resembles Equation (21) when the equilibrium timescale $T_{e q}=7.1 \times 10^{6}$ yrs is chosen as the moment when the smallest dust have reached two-thirds their equilibrium abundance. This demonstrates that the two-component dust size model of Section 2.4.1 is in fact relevant to this kind of debris disk. And because $T_{0}$ and $T_{e q}$ have the same dependance on the model parameters, comparison to Equation (28c) shows that $T_{e q} \simeq 130 T_{0}$.

After a time $t \gg T_{e q}$, the debris disk will have settled into equilibrium. The solid black curve in Figure 3 shows the disk's equilibrium grain size distribution $N(R)$, which is extracted from the rightmost part of Figure 2 and plotted versus grain radii $R$. That model assumes that the dust grains are weak, $Q^{\star}<10^{6} \mathrm{ergs} / \mathrm{gm}$, which means that all collisions are destructive. Note that the debris disk's dust size distribution $N(R)$ is significantly steeper 
than the $q=3.5$ size distribution that governs the ring's dust production (the dotted curve in Figure [3), with $N(R) \propto R^{-6}$ except near $R \simeq R_{\text {min }}$, where it has an even steeper dependance. A peak at $R \simeq R_{\text {min }}$ is also seen in the debris disk mode 2 of Krivov et al. (2006). Figure 3 illustrates the main consequence of dust-dust collisions, which tends to destroy the disk's larger dust grains at a faster pace. However, when the dust grains are stronger, with $10^{7}<Q^{\star}<10^{8} \mathrm{ergs} / \mathrm{gm}$, then the larger grains are more resistant to collisional destruction, and they become more abundance (Figure 3 , grey curves).

When the system is in equilibrium, the rate at which the planetesimal ring injects dust of size $\beta_{i}$ into the debris disk, $P_{i}$, balances the rate at which collisions remove dust from the disk $\mathcal{R}_{i}$, so $\mathcal{R}_{i}=P_{i}$. Thus the collisional lifetime of the grains in streamline $i$ is $T_{c}\left(R_{i}\right)=N_{i} / \mathcal{R}_{i}=N_{i} / P_{i}$, which is plotted versus grain radius $R$ in Figure 4 (see the black curves that are labeled by their dust production rates $\dot{M}_{d}$ ). That lifetime is simply the time when the dust abundances $N_{i}(t)$ seen in Figure 2 flatten out. As expected, the smallest grains that have size parameters $\beta$ that are just shy of $\beta_{\max }=\frac{1}{2}$ are very long lived. This is due to their orbits having very large apoapses $Q_{\text {apo }}=r_{\text {out }} /(1-2 \beta) \gg r_{\text {out }}$, so small dust spend most of their time far from the planetesimal ring, in regions where the disk's optical depth is low and collisions are rare. Note also that $T_{c} \propto\left(N_{0} / P_{0}\right) \propto T_{0}$, so the dust grains' collision timescale obeys the same scaling as Equation (28c), in particular with $T_{c} \propto \dot{M}_{d}^{-1 / 2}$. Figure 4 also shows that nearly all dust grains in such a debris disk have lifetimes $T_{c}$ that are short compared to the age of the host star, which is typically $\sim 10^{7}$ to $10^{8}$ years (Meyer et al. 2007).

When the grains are weak, $Q^{\star}<10^{6} \mathrm{ergs} / \mathrm{gm}$, then the lifetime of the larger $R \gtrsim$ $2 R_{\min }$ varies at $T_{c}(R) \propto R^{-2.4}$ (Figure 4). That figure also shows that increasing the dust grains' strength $Q^{\star}$ increases the larger grains' longevity, without affecting the small grains' collisional lifetime. This is because all collisions are energetic enough to destroy all small grains.

\footnotetext{
${ }^{2}$ Note though that the equilibrium dust size distribution in the Krivov et al. (2006) model is 'wavy'. Waves in a size-distribution occur when some process tends to favor the rapid removal of the smallest bodies (Durda \& Dermott 1997), and Figure 5 of Krivov et al. (2006) would suggest that next peak in our size distribution might occur at sizes $R \sim 1 \mathrm{~mm}$. However these large grains would still have a very short collisional lifetime (see Figure 4), which makes their influence in the dust-disk quite negligible.
} 


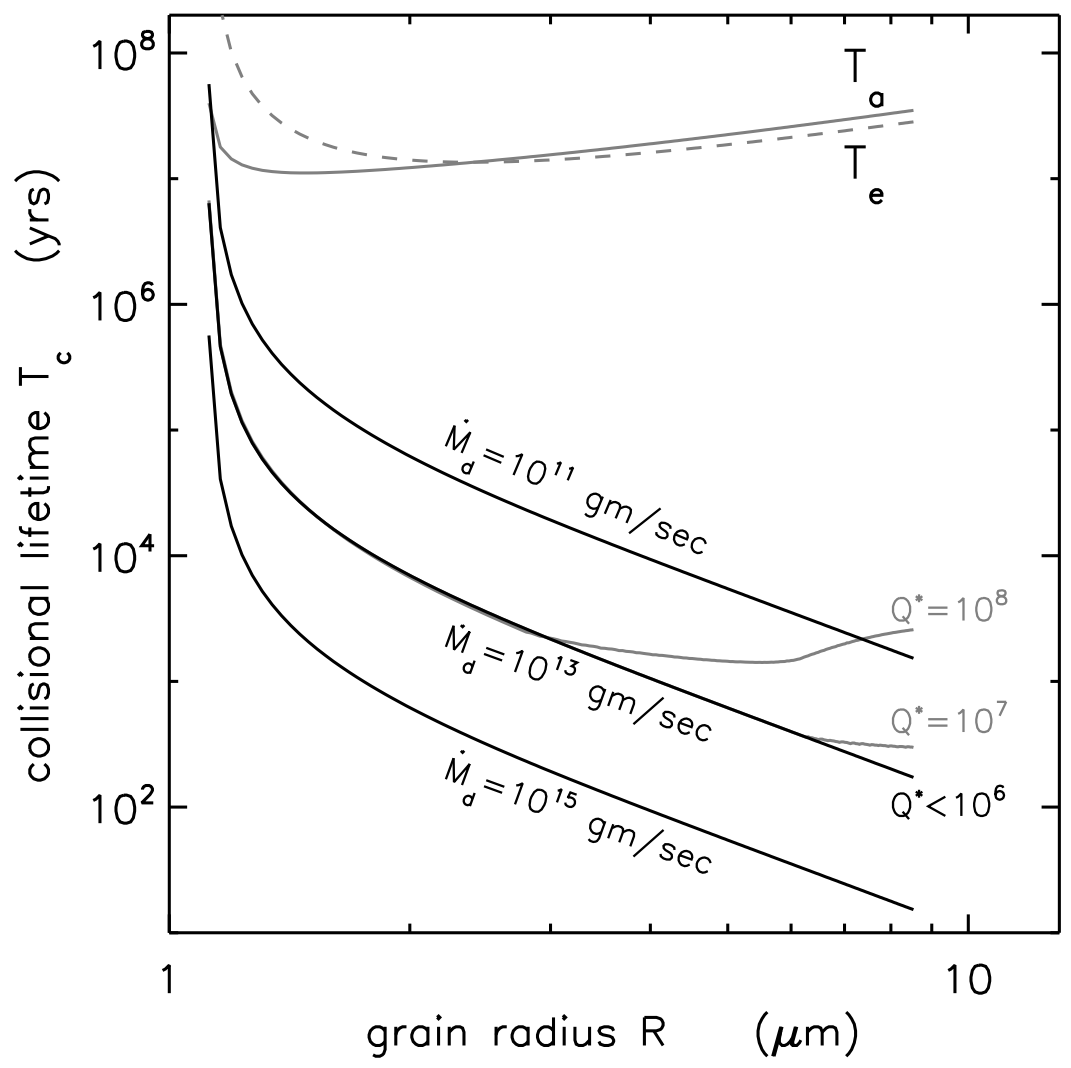

Fig. 4.- The lower middle black curve is the dust grain's collisional lifetime $T_{c}(R)$ plotted versus dust radius $R$ for the debris-disk model of Figure 2 that has a dust production rate $\dot{M}_{d}=10^{13} \mathrm{gm} / \mathrm{sec}$ and strength $Q^{\star}<10^{6} \mathrm{ergs} / \mathrm{gm}$, while the other black curves are for systems that are otherwise identical but with the indicated dust production rates $\dot{M}_{d}$. The grey curves are for stronger dust grains having $Q^{\star}=10^{7}$ and $10^{8} \mathrm{ergs} / \mathrm{gm}$ and $\dot{M}_{d}=10^{13}$ $\mathrm{gm} / \mathrm{sec}$; compare also to Figure 3. The upper solid grey curve $T_{a}$ is the timescale over which the dust grains' semimajor decays due to PR drag, with $T_{e}$ the timescale for eccentricity damping due to PR drag; see Equations (36).

\subsection{4. optical depth}

To determine the model debris disk's normal optical depth $\tau_{n}(r)$, it is convenient to first calculate the total dust cross section $A(r)$ that resides interior to distance $r$ from the star. That quantity is obtained by first counting the number of dust grains $\Delta n_{j}(r)$ in streamline $j$ that also lie interior $r$, which is $\Delta n_{j}(r)=\int \lambda_{j} d \ell$ where the integration runs along the stretch of streamline interior to $r$. This becomes a trivial integral over time $t$ after noting $\lambda_{j} d \ell=\lambda_{j} v_{j} d t$ where $\lambda_{j}=n_{j} / v_{j} T_{j}$ is the dust grain's linear density, and $n_{j}$ is the total number of grains in streamline $j$ that have velocity $v_{j}$ and orbit period $T_{j}$. Consequently, $\Delta n_{j}(r)=$ $2 n_{j} t_{j}(r) / T_{j}$ where $t_{j}(r)$ is the time for dust in streamline $j$ to travel from periapse to distance $r$. And since $n_{i}=N_{i} / N_{l}$ where $N_{i}$ is the total number of dust grains in the disk that have 


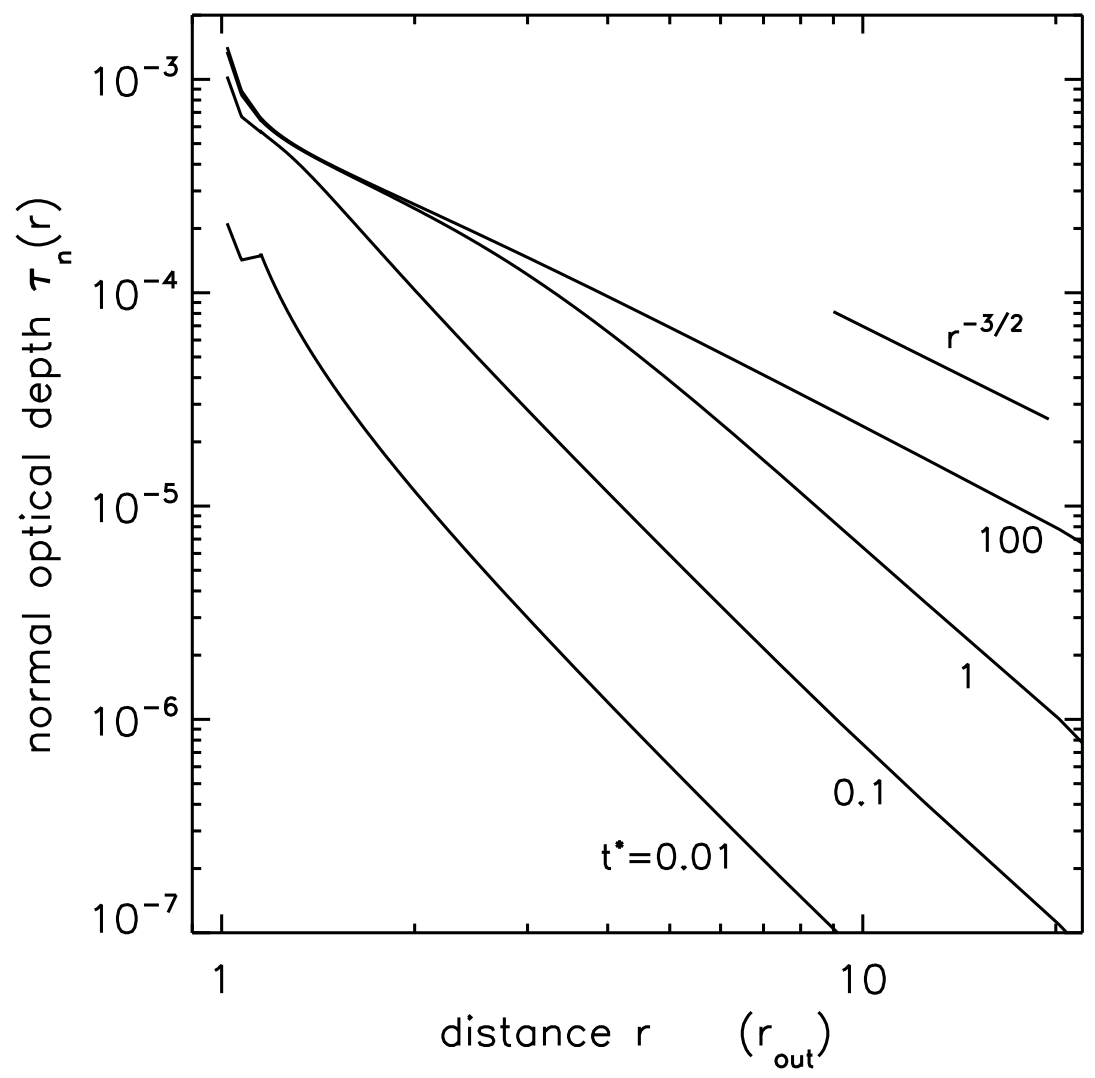

Fig. 5.- The dust optical depth $\tau_{n}(r)$ is plotted versus distance $r$ in units of the planetesimal disk's outer radius $r_{\text {out }}$ for the model of Figure 2. Shown is $\tau_{n}(r)$ at dimensionless times $t^{\star}=0.01,0.1,1,100$, and an $r^{-3 / 2}$ curve.

the same sizes and orbits, the total cross section for grains of radii $R_{i}=\left(\beta_{\max } / \beta_{i}\right) R_{\min }$ that reside interior to $r$ is $A_{i}(r)=N_{l} \Delta n_{i}(r) \pi R_{i}^{2}=2 N_{i}\left(t_{i} / T_{i}\right)\left(\beta_{\max } / \beta_{i}\right)^{2} \pi R_{\text {min }}^{2}$. The total dust cross section interior to $r$ is then $A(r)=\sum_{i} A_{i}(r)$ where the sum proceeds over all streamlines whose dust have distinct sizes $\beta_{i}$ produced by planetesimal ring $r_{i}$. The ratio $t_{i} / T_{i}$ in the above is obtained by solving $r=a_{i}\left(1-e_{i} \cos E\right)$ for the dust grains' eccentric anomaly $E(r)$, which is then inserted into Kepler's equation to obtain $t_{i} / T_{i}=\left(E-e_{i} \sin E\right) / 2 \pi$. Also note that the differential $\Delta A=(\partial A / \partial r) \Delta r$ is the dust cross section that resides in an annulus of radius $r$ and width $\Delta r$. Since the disk's normal optical depth $\tau_{n}$ is simply the surface density of dust cross section, that quantity is

$$
\tau_{n}(r)=\frac{\Delta A}{2 \pi r \Delta r}=\frac{1}{2 \pi r} \frac{\partial A}{\partial r}
$$

which is easily calculated by differentiating $A(r)$ numerically.

Figure 5 plots the dust optical depth $\tau_{n}(r)$ versus distance $r$ from the star at dimensionless times $t^{\star}=0.01,0.1,1$, and 100 for the model of Figure 2. As the figure shows, it is the outer portion of the disk that is populated at later times by dust. This is due to the 


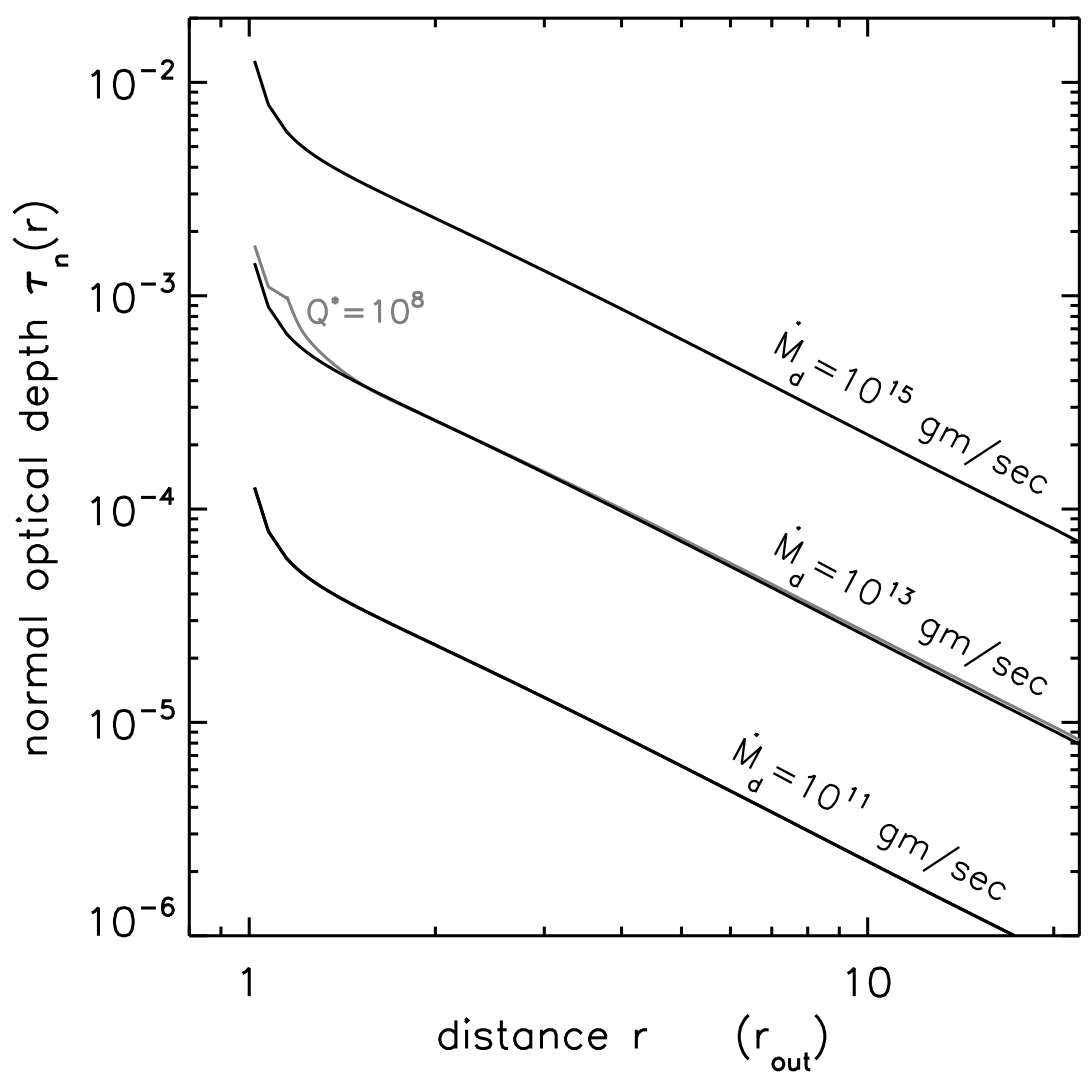

Fig. 6.- Black curves give the debris disk's equilibrium optical depth $\tau_{n}(r)$ versus radial distance $r$ for dust generated in a narrow debris disk of radius $a_{r}$. Model parameters are identical to those adopted in Figure 2 except that different dust production rates are considered, $\dot{M}_{d}=10^{11}, 10^{13}$, and $10^{15} \mathrm{gm} / \mathrm{sec}$, and weak grains having $Q^{\star}<10^{6} \mathrm{ergs} / \mathrm{gm}$, while the one grey curve assumes strong dust having $Q^{\star}=10^{8} \mathrm{ergs} / \mathrm{gm}$.

outer disk being composed of smaller dust that, according to Figure 2, are the last to arrive at a collisional balance. Also note that when the disk has settled into equilibrium at times $t^{\star} \gg 1$, the outer disk at $r \gg r_{\text {out }}$ has an optical depth $\tau_{n}(r) \propto r^{-3 / 2}$, which is in agreement with what Strubbe \& Chiang (2006) call a type B debris disk.

The black curves in Figure [6 show the equilibrium optical depth $\tau_{n}(r)$ for simulated debris disks that are identical to the one considered in Figure 2, except that different dust production rates $\dot{M}_{d}$ are considered. These curves assume the dust grains are weak, with $Q^{\star}<10^{6} \mathrm{ergs} / \mathrm{gm}$. As expected, these optical depths vary as $\dot{M}_{d}^{1 / 2}$ (see Equation 28a). The one grey curve there shows the optical depth of a disk composed of strong dust having $Q^{\star}=10^{8} \mathrm{ergs} / \mathrm{gm}$. That disk shows a slight overdensity near the planetesimal ring, and is due to an excess of larger grains in low-eccentricity orbits (see grey curves in Figures 3 and (4). 


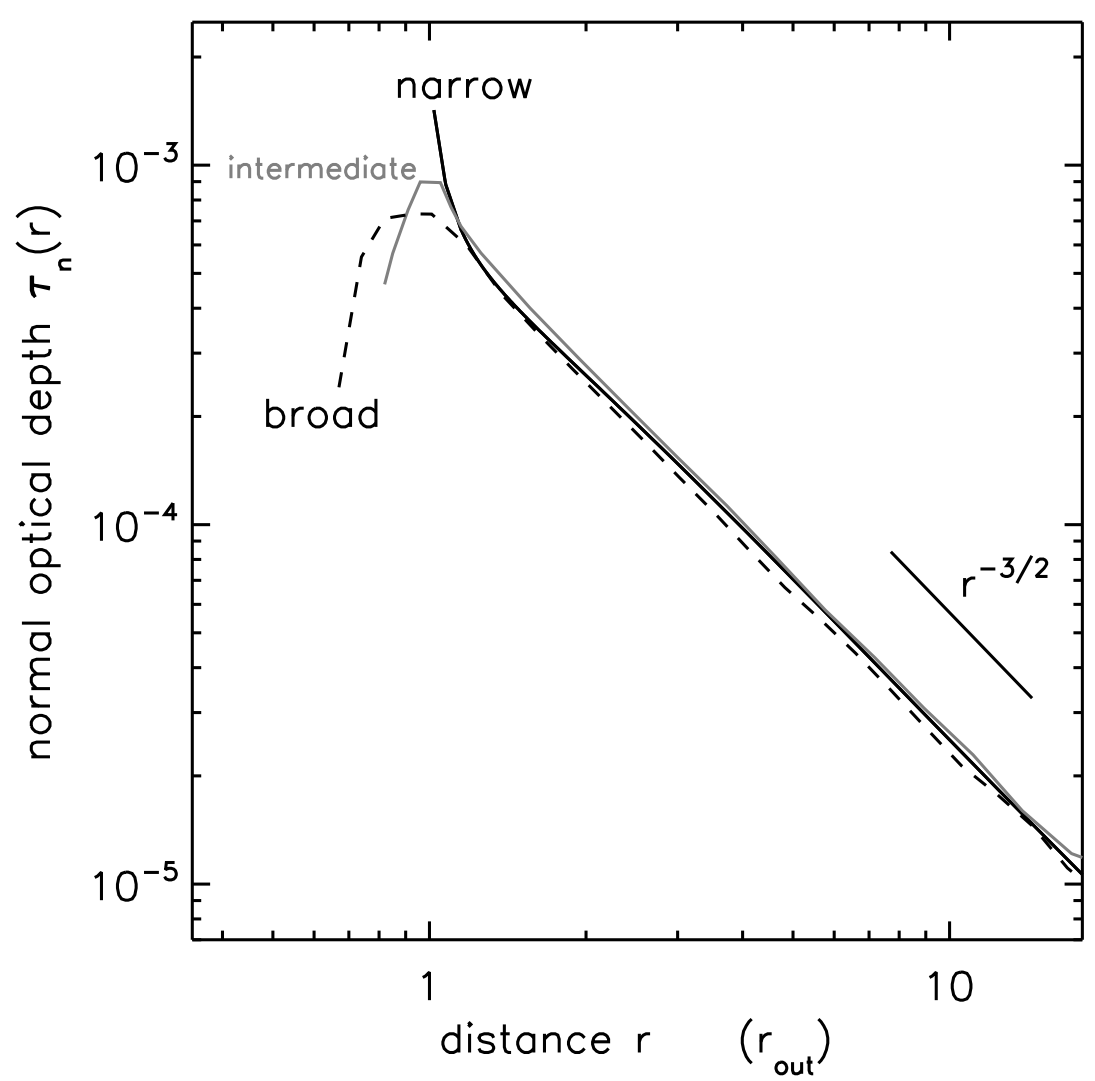

Fig. 7.- The normal optical depth $\tau_{n}(r)$ for three debris disks. One is generated by a narrow planetesimal ring of radius $r_{\text {out }}$ (black curve), another by an intermediate-width planetesimal ring whose inner radius $r_{i n}=0.8 r_{\text {out }}$ (grey curve), and the third due to a broad planetesimal disk having $r_{\text {in }}=0.5 r_{\text {out }}$ (dashed curve). The narrow planetesimal disk is represented by one planetesimal ring $\left(N_{r}=1\right)$, while the intermediate and broad disks use $N_{r}=3$ and $N_{r}=5$ planetesimal rings, respectively. All simulations have $c=0$, so the dust production rate is independent of distance $r$ in the planetesimal disk. Model parameters are otherwise identical to those adopted in Figure 2. Also shown is an $r^{-3 / 2}$ curve.

The sharp peak in $\tau_{n}(r)$ at $r \simeq r_{\text {out }}$ seen in Figure [6 is due to the narrow width of the planetesimal ring that is the source of this dust. However, Figure 7 shows that peak broadens when the planetesimal ring's radial width is increased.

\subsection{5. disk surface brightness}

This subsection will calculate the surface brightness of starlight that a simulated debris disk will scatter towards an observer that views the disk at optical wavelengths. In the following, flux $F$ is the power per area in the incident or scattered radiation, intensity $I$ is 

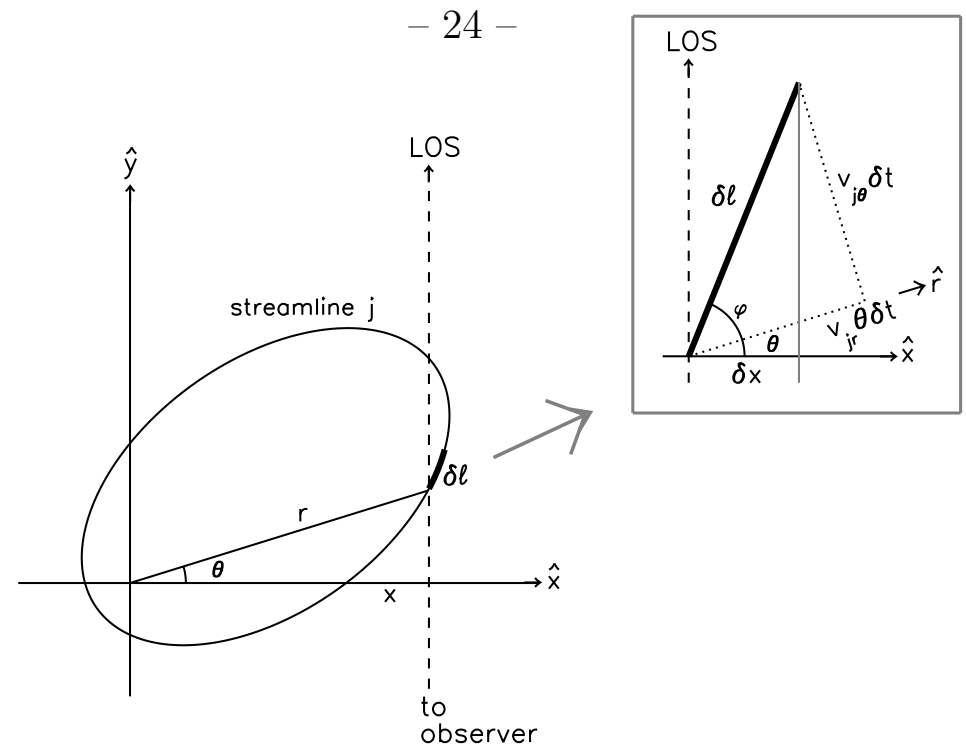

Fig. 8.- The observer's line-of-sight (LOS) passes through streamline $j$ and intersects segment $\delta \ell$ at polar coordinates $(r, \theta)$; that segment also lies at a projected distance $x$ from the central star at the origin. The insert zooms in on segment $\delta \ell$, which has a projected length $\delta x$ when viewed by the observer. The dust in that segment will have traveled a distance $\delta \ell=v_{j} \delta t$ in time $\delta t$, which corresponds to radial and tangential displacements $v_{j, r} \delta t$ and $v_{j, \theta} \delta t$, so the angle $\varphi$ satisfies $\sin \varphi=v_{j, \theta} / v_{j}$ and $\cos \varphi=v_{j, r} / v_{j}$. Also note that the scattering angle $\phi$ is the angle between the radial direction $\hat{\mathbf{r}}$ and the direction to the observer, so $\phi=\pi / 2+\theta$.

the power per solid angle in a pencil-beam of radiation, and the surface brightness $B$ is the radiation beam's power per area per solid angle.

Begin by calculating the surface brightness of a small segment of length $\delta \ell_{j}$ in streamline $j$. Summing the contributions from all such streamlines will then provide the debris disk's total surface brightness along some line-of-sight. Since these disks are often observed nearly edge on, an edge-on viewing geometry is also assumed here, though these results are easily generalized for other viewing geometries as well. Segment $\delta \ell_{j}$ in streamline $j$ is composed of dust having a total cross section $\delta \sigma_{j}$, so the intensity of starlight $\delta I_{j}$ that is reflected by that segment is $\delta I_{j}=Q_{s} \Phi F_{i} \delta \sigma_{j}$, where $F_{i}=L_{\star} / 4 \pi r^{2}$ is the flux of incident starlight, $L_{\star}$ is the central star's luminosity, and $r$ the dust grains' distance from the star. The dust grains' phase function is $\Phi$, and it is normalized so that its integral over all solid angles is unity, with this quantity having units of steradians ${ }^{-1}$. Note also that $F_{i} \delta \sigma$ is the power in the incident starlight while $\int \delta I_{j} d \Omega$ (when integrating over all solid angles $d \Omega$ ) is the power in the scattered light, so $Q_{s}=\int \delta I_{j} d \Omega / F_{i} \delta \sigma_{j}$ is the efficiency of light scattering by these dust grains. In planetary astronomy this quantity is known as the bond albedo (Lester et al. 1979). 
The surface brightness of the small segment is $B_{j}=\delta I_{j} / \delta A_{j}$ where $\delta A_{j}$ is the projected area on the sky occupied by that segment whose total length is $\delta \ell_{j}$ and projected length (as seen by the observer) is $\delta x_{j}$; see Figure 8. Since that segment is actually a ribbon of material of height 2 Ir due to the dust grain's inclinations $I$, the segment's projected area is $\delta A_{j}=2 \operatorname{Ir} \delta x_{j}$, and its surface brightness contribution is $B_{j}=\left(Q_{s} \Phi F_{i} / 2 \operatorname{Ir}\right)\left(\delta \sigma_{j} / \delta x_{j}\right)$.

The number of dust grains in segment $\delta \ell_{j}$ is $\delta n_{j}=\lambda_{j} \delta \ell_{j}=\lambda_{j} v_{j} \delta t$ where $v_{j}$ is the dust grains' velocity there and $\delta t$ is the time for the dust to traverse $\delta \ell_{j}$. But this becomes $\delta n_{j}=\left(n_{j} / T_{j}\right) \delta t$ where $T_{j}$ is the dust grains' orbit period, since $\lambda_{j} v_{j}=n_{j} / T_{j}$ where $n_{j}$ is the number of dust grains in streamline $j$ that also have radius $R_{j}=\left(\beta_{\max } / \beta_{j}\right) R_{\min }$. Consequently, the cross section of dust in segment $\delta \ell_{j}$ is $\delta \sigma_{j}=\pi n_{j}\left(\beta_{\max } / \beta_{j}\right)^{2} R_{\text {min }}^{2}\left(\delta t / T_{j}\right)$. That segment has length $\delta \ell_{j}=v_{j} \delta t$, and Figure 8 shows that its projected length is $\delta x_{j}=$ $v_{j}|\cos (\theta+\varphi)| \delta t$ where $\theta$ is the segment's longitude as measured from the $\hat{\mathbf{x}}$ axis. The angle $\varphi$ obeys $\sin \varphi=v_{j, \theta} / v_{j}$ and $\cos \varphi=v_{j, r} / v_{j}$ (see Figure 8) where $v_{j, r}$ and $v_{j, \theta}$ are the dust grain's radial and tangential velocities, Equations (13), so $\delta x_{j}=\left|v_{j, r} \cos \theta-v_{j, \theta} \sin \theta\right| \delta t=$ $2 \pi a_{j}\left|\sin \theta+e_{j} \sin \tilde{\omega}_{j}\right|\left(\delta t / T_{j}\right) / \sqrt{1-e_{j}^{2}}$. Inserting these results into $B_{j}$ and noting that $n_{j}=$ $N_{j} / N_{l}$ then yields $B_{j}=B_{0} \sqrt{1-e_{j}^{2}}\left(\Phi \Omega_{1} N_{j} / N_{l}\right)\left(R_{\text {min }}^{2} r_{\text {out }}^{2} / r^{3} a_{j}\right)\left(\beta_{\text {max }} / \beta_{j}\right)^{2} /\left|\sin \theta+e_{j} \sin \tilde{\omega}_{j}\right|$ where the constant

$$
B_{0}=\frac{Q_{s} L_{\star}}{16 \pi I r_{\text {out }}^{2} \Omega_{1}}
$$

Note that $\Omega_{1}=1$ steradian is introduced into the above so that $B_{0}$ has units of surface brightness and that the combination $\Phi \Omega_{1}$ is dimensionless. Summing the contributions from all streamlines then yields the disk's total surface brightness $B(x)=\sum B_{j}$ as a function of projected distance $x=r \cos \theta$ from the central star, so

$$
B(x)=B_{0} \sum_{i=1}^{N_{r \beta}} \sum_{j=1}^{N_{l}} \sum_{\text {near }}^{\text {far }} \frac{\Phi \Omega_{1} N_{i} \sqrt{1-e_{i}^{2}}}{N_{l}\left|\sin \theta+e_{j} \sin \tilde{\omega}_{j}\right|}\left(\frac{R_{\text {min }}}{r_{\text {out }}}\right)^{2}\left(\frac{r_{\text {out }}^{4}}{r^{3} a_{i}}\right)\left(\frac{\beta_{\text {max }}}{\beta_{i}}\right)^{2}
$$

where the innermost sum is over the two segments - one nearer and the other further from the observer - that intersect the observer's line-of-sight (see Figure 8), while the middle sum proceeds over each streamlines' orientation $\tilde{\omega}_{j}$, and the leftmost sum proceeds over the $N_{r \beta}$ streamlines having distinct dust sizes $\beta_{i}$ that originate in the various planetesimal rings $r_{i}$. Equation (31) also requires the two longitude $\theta(x)$ where the observer's line-of-sight intercepts streamline $j$. That is obtained by solving $x=r \cos \theta$ for $\theta(x)$ where $r=p_{j} /\left[1+e_{j} \cos \left(\theta-\tilde{\omega}_{j}\right)\right]$, which yields $\theta(x)=\tilde{\omega}_{j}-\tan ^{-1}(B / A) \pm \cos ^{-1}\left(x / \sqrt{A^{2}+B^{2}}\right)$ where $A=p_{j} \cos \tilde{\omega}_{j}-e_{j} x$, $B=p_{j} \sin \tilde{\omega}_{j}$, and $p_{j}=a_{j}\left(1-e_{j}^{2}\right)$ is the ellipse's semi-latus rectum, with the $r$ in Equation (31) from $r=x / \cos \theta$. 


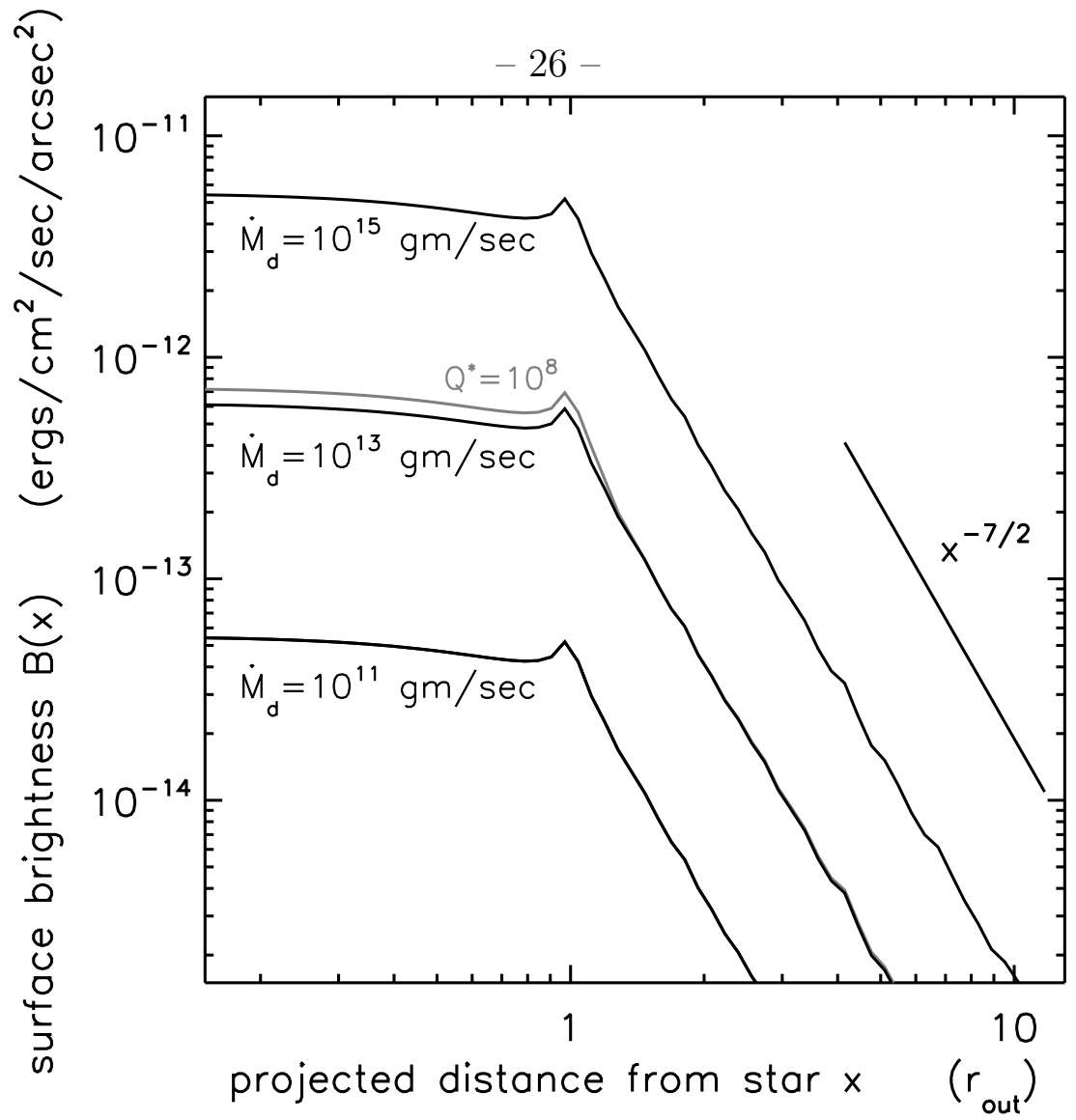

Fig. 9.- Equation (31) is used to calculate the surface brightness $B(x)$ versus projected distance from the star $x$ for three edge-on debris disks that are generated by narrow planetesimal rings that have the indicated dust production rates $\dot{M}_{d}=10^{11}, 10^{13}$, and $10^{15} \mathrm{gm} / \mathrm{sec}$. Black curves assume weak dust having $Q^{\star}<10^{6} \mathrm{ergs} / \mathrm{gm}$, a light scattering asymmetry parameter $g=0.4$ and albedo $Q_{s}=0.1$, with all system parameters identical to those of Figure 2, These curves give the disks' unfiltered surface brightnesses integrated over all optical wavelengths. A $x^{-7 / 2}$ curve is also shown, and the grey curve is for strong dust having $Q^{\star}=10^{8} \mathrm{ergs} / \mathrm{gm}$.

The phase function employed here is the Henyey-Greenstein function

$$
\Phi(\phi)=\frac{1-g^{2}}{4 \pi\left(1+g^{2}-2 g \cos \phi\right)^{3 / 2}} \quad \operatorname{ster}^{-1}
$$

that is widely used in studies of circumstellar dust. The scattering angle $\phi$ in the above is related to the dust grain's longitude $\theta$ via $\phi=\pi / 2+\theta$; see Figure 8. This phase function is controlled by the dust grains' asymmetry parameter $g=\int \Phi(\phi) \cos \phi d \Omega$ (see Equation 2), with a positive value resulting in forward light scattering while negative values result in backscattering.

Equations (31-32) are used to calculate the surface brightness for three edge-on debris disks whose parameters are identical to those of Figure 2 except for differing dust production 


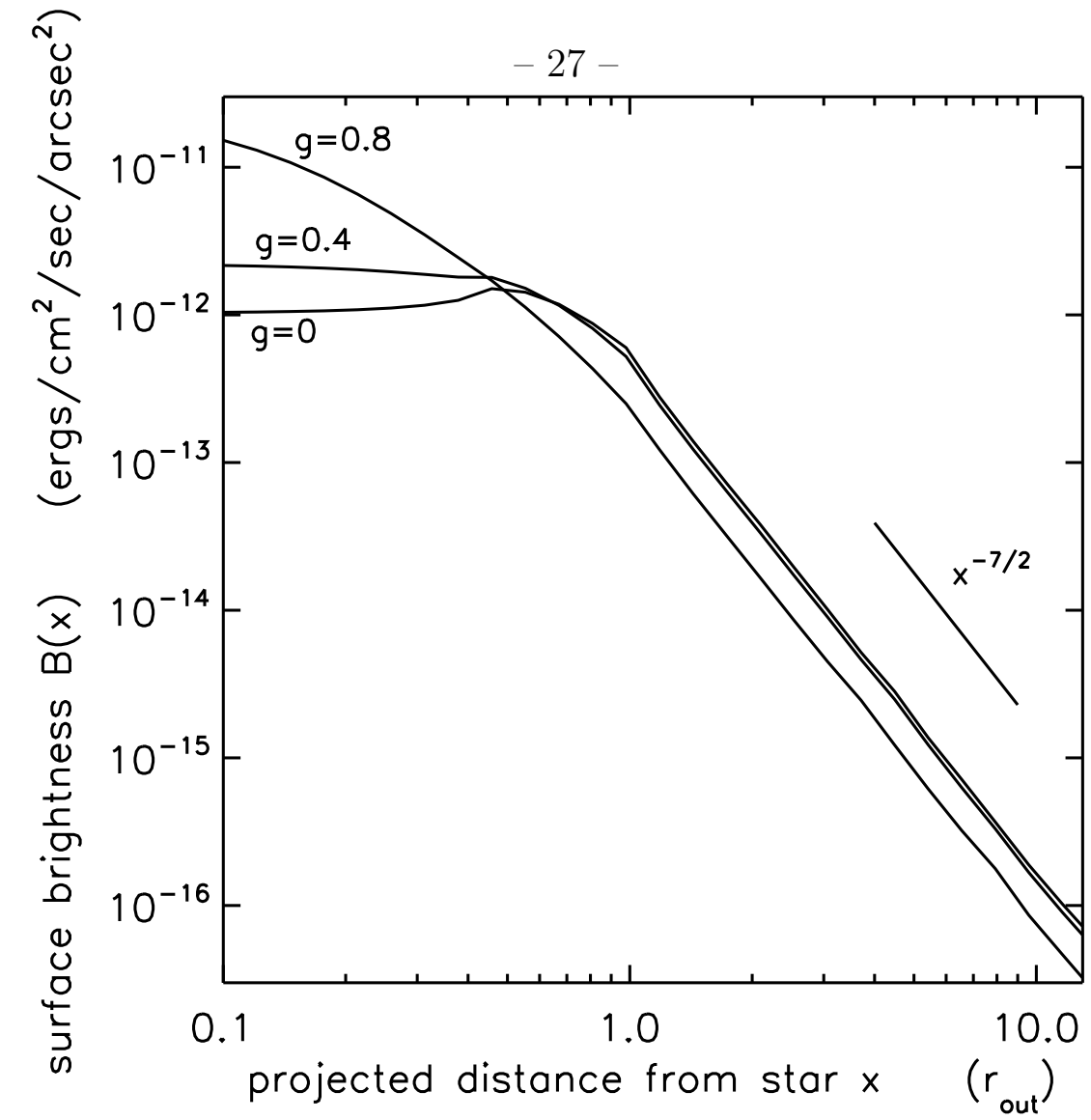

Fig. 10. - Surface brightness $B(x)$ is plotted versus projected distance $x$ for three edge-on debris disks that are generated by a broad $\left(r_{\text {in }}=0.5 r_{\text {out }}\right)$ planetesimal disk having a dust production rate $\dot{M}_{d}=10^{13} \mathrm{gm} / \mathrm{sec}$, a light scattering efficiency of $Q_{\text {scat }}=0.1$, and the indicated light scattering asymmetry parameter $g=0,0.4$, and 0.8 . Also shown is an $x^{-7 / 2}$ curve. Note also that the small bump in $B(x)$ at $x=0.5 r_{\text {out }}$ (which is where the line-of-sight runs along the planetesimal disk's inner edge) gets washed-out when the light scattering is very asymmetric with $g \gtrsim 0.8$.

rates $\dot{M}_{d}$; see Figure 9. As expected, these surface brightness curves vary as $B \propto \sqrt{\dot{M}_{d}}$. Also note that $B(x) \propto x^{-7 / 2}$, which again is in agreement with the type $\mathrm{B}$ debris disk of Strubbe \& Chiang (2006).

Figure 10 illustrates how the inner part of a debris disk's surface brightness depends on the degree of asymmetry in the dust grains' light scattering. For instance, when the dust grains are either isotropic light scatters $(g=0)$, or are only modestly forward scattering $(g=0.4)$, the debris disk's surface brightness profile $B(x)$ is roughly constant in the inner regions where $x \lesssim r_{\text {out }}$. However, if the light scattering by the dust is strongly asymmetric, with $g=0.8$, then $B(x)$ continues to increase inwards of $x=r_{\text {out }}$. Disks having this kind of knee-bend in their surface brightness profiles appear to be common (Krist et al. 2005; Golimowski et al. 2006), with forward scattering of starlight being the preferred explanation 
for the bent surface brightness profile of the edge-on debris disk at AU Mic and $\beta$ Pic (Strubbe \& Chiang 2006; Ahmic et al. 2009). Evidently, light scattering by circumstellar dust is rather asymmetric. Lastly, note that almost identical results are obtained when the dust grains are backscattering, with $g<0$, with those slight differences being due to the dust grains' slightly larger radiation pressure efficiency $Q_{r p}=1-g Q_{s}$. And the following will assume that the grains are forward scattering, which is appropriate for dust grains that are larger than the wavelength of the incident radiation, and is consistent with forward scattering by the Solar System's interplanetary dust (Lamy \& Perrin 1986).

Figure 11 also shows how an edge-on disk's surface brightness $B(x)$ evolves over time. As expected, $B(x)$ gets shallower at later times as the smaller dust grains steadily populate the outer parts of the disk; see also Figures 2 and 5. Eventually, the smaller grains arrive at collisional equilibrium at dimensionless times $t^{\star}=t / T_{0} \gg 1$, and the disk surface brightness settles into the expected $B(x) \propto x^{-3.5}$ power law. But note that at earlier times, such as at time $t^{\star}=0.01$, the disk's surface brightness can be as steep as $B(x) \propto x^{-5.0}$. Consequently, disks having a surface brightness steeper than $x^{-3.5}$ might indicate that the disk is younger than time $T_{0}$, or that dust production by the planetesimal disk has increased in recent times.

Note that these surface brightness calculations assume that the dusty disk is so tenuous that the grains do not shadow each other; this assumption is confirmed in Section 3.3.2. Also keep in mind that Figures 911 give the disks' total surface brightness integrated over all optical wavelengths. If these systems were instead observed through a narrowband filter having a central frequency $\nu$ then the star's total luminosity $L_{\star}=4 \pi^{2} R_{\star}^{2} \int_{0}^{\infty} B_{\nu} d \nu=4 \pi R_{\star}^{2} \sigma T_{\star}^{4}$ (where $R_{\star}$ is the stellar radius, $B_{\nu}$ is the Planck function, $\sigma$ the Stefan-Boltzmann constant, and $T_{\star}$ is the star's effective temperature) in Equation (30) should instead be replaced by its specific luminosity $\partial L_{\star} / \partial \nu=\pi L_{\star} B_{\nu} / \sigma T_{\star}^{4}$, so that Equations (31 32) would then provide the disk's surface brightness per unit frequency.

\subsection{6. a relic debris disk}

It is also worth considering the debris disk's evolution when the planetesimal disk's dust production suddenly ceases. Such might occur if there are any planets in the system that quickly adjust their orbits, perhaps due to a rapid migration of a planet through a dense planetesimal disk (Gomes et al. 2004), or due to planet-planet scattering (i.e., the Nice model, Gomes et al. 2005). Should that occur, then the planetesimals might find themselves in unstable orbits, which then leads to a rapid dynamical erosion of the disk and a cessation of dust production. 


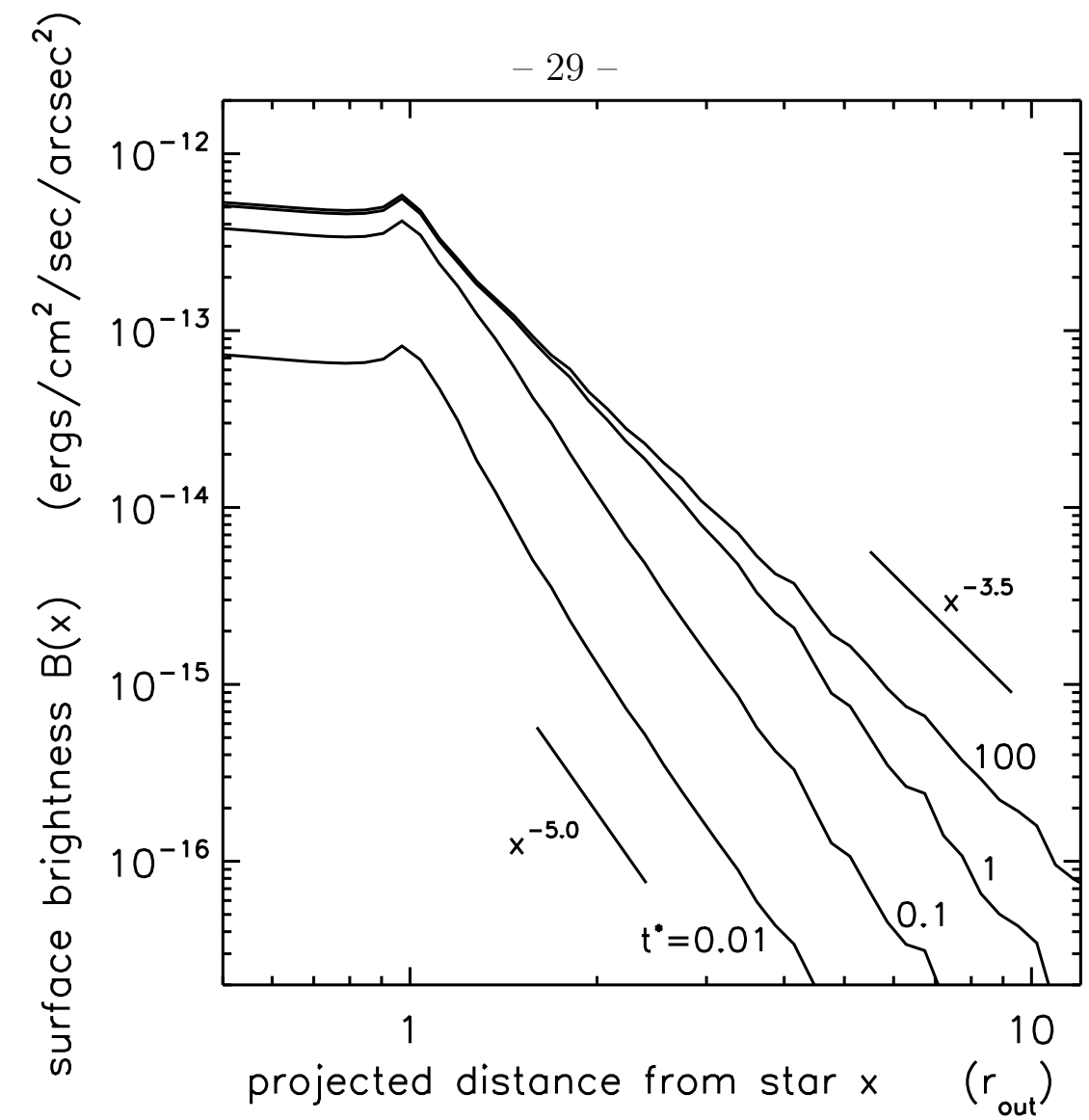

Fig. 11. - Surface brightness $B(x)$ is plotted versus projected distance $x$ (in units of $r_{\text {out }}$ ) for the debris-disk model that is described in Figures 2$]$ and 5 viewed edge-on with $Q_{s}=0.1$. Shown is $B(x)$ at dimensionless times $t^{\star}=t / T_{0}=0.01,0.1,1$, and 100 , as well as power laws that vary as $x^{-3.5}$ and $x^{-5.0}$.

Halting dust production is simulated here by setting the planetesimal ring's dust production rate $P_{i}(t)=0$ at all times after $t_{\text {stop }}$, or equivalently changing the $1 \rightarrow 0$ in the invariant evolutions equation (27) at dimensionless times $t^{\star}>t_{\text {stop }}^{\star}$ where $t_{\text {stop }}^{\star}=t_{\text {stop }} / T_{0}$. Solving those equations numerically shows that the larger grains in the inner part of the debris disk are quickly destroyed due to their short collisional lifetimes (Figure 41). This depletes the inner disk's optical depth and drives it towards a shallower $\tau_{n}(r) \propto r^{-1 / 2}$ power law that decreases over time. And as Figure 12 shows, the cessation of dust production also decreases the inner part of an edge-on disk's surface brightness so that $B(x) \propto x^{-5 / 2}$ at times $t^{\star} \gg t_{\text {stop }}^{\star}$. So the detection of a debris disk having a shallow optical depth profile $\tau_{n}(r) \propto r^{-1 / 2}$ (when the view to the disk is oblique or face-on), or an edge-on disk having a shallow $B(x) \propto x^{-5 / 2}$ surface brightness profile, would indicate that the object is a relic disk wherein dust-production has ceased. That relic disk will be composed mostly of small, marginally-bound grains of radii $R \simeq R_{\text {min }}$. The abundance $N_{S}$ of those small dust grains will then fade over time according to Equation (20), whose solution is $N_{S}\left(t^{\prime}\right)=N_{S}\left(t_{\text {stop }}\right) /\left[1+N_{S}\left(t_{\text {stop }}\right) \bar{\alpha}_{S S} t^{\prime} / T_{\text {out }}\right]$ when the 


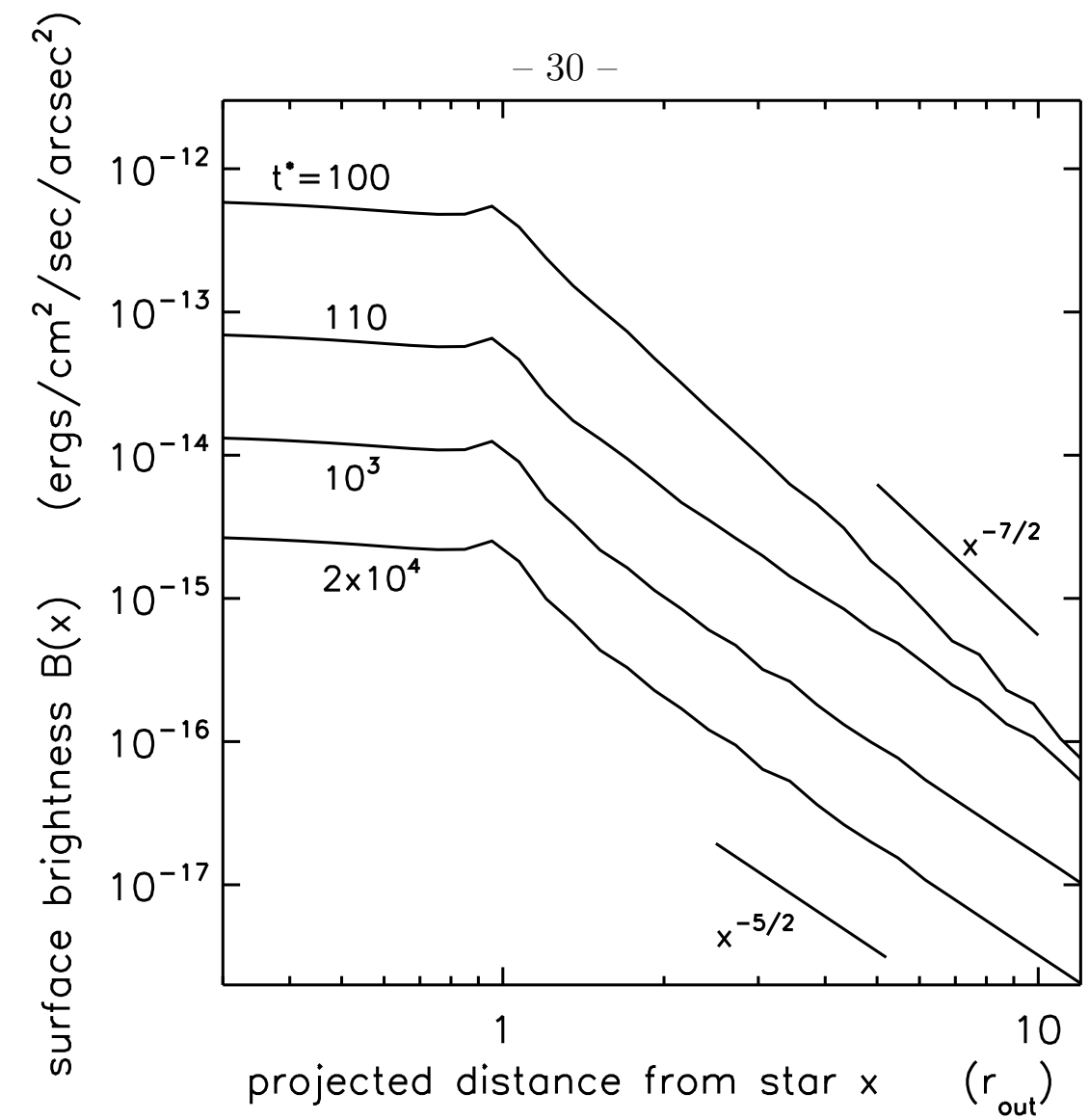

Fig. 12.- The dust-producing debris disk model of Figure 2 is evolved until dimensionless time $t_{\text {stop }}^{\star}=100$ when dust production ceases $\left(P_{i}=0\right)$. The debris disk's surface brightness $B(x)$ is shown at the indicated times $t^{\star}$, as well as two power laws $x^{-7 / 2}$ and $x^{-5 / 2}$.

dust production rate $P_{S}=0$, where $t^{\prime}=t-t_{\text {stop }}$ is the time since the end of dust production and $\bar{\alpha}_{S S}$ is the small grains' collisional probability density. Note that this behavior over time is typical for a system that steadily grinds away without any replenishment ( $c f$., Wyatt et al. 2007a; Löhne et al. 2008). Inspection of the upper two curves in Figure 12 also shows that the time for the system to transform into a relic disk is about $t_{\text {trans }} \sim 10 T_{0} \sim 0.1 T_{\text {eq }}$ i.e., the transition occurs relatively quickly in comparison to the system's equilibrium timescale $T_{e q}$.

\section{Application to $\beta$ Pictoris}

The colored curves in Figure 13 show the surface brightness of the starlight that is scattered by the edge-on debris disk orbiting $\beta$ Pictoris. These curves are extracted from images that Golimowski et al. (2006) acquired with the Hubble Space Telescope (HST) at optical wavelengths. These profiles exhibit the classic signature of a debris disk that is generated by a disk of colliding planetesimals that extends out to about $r_{\text {out }} \simeq 150 \mathrm{AU}$, 
with the surface brightness falling off steeply as $B(x) \propto x^{-3.5}$ at projected distances $x \gtrsim 220$ $\mathrm{AU}$, and less steep interior to $r_{\text {out }}$. Note also that the $\beta$ Pic disk is asymmetric, with the outer part of the disk's northeast (NE, blue curve) ansa being about $50 \%$ brighter than its southwest (SW, red curve) ansa. Although that asymmetry is not accounted for by the axially symmetric debris-disk model that is used here, possible causes for that asymmetry are described in Section 3.3.5.

$\beta$ Pic is an A5V star that lies a distance $\Delta=19.28$ pc away, has a luminosity $L_{\star}=8.7 \mathrm{~L}_{\odot}$, mass $M_{\star} \simeq 1.8 \mathrm{M}_{\odot}$, an effective temperature $T=8200 \mathrm{~K}$ (Crifo et al. 1997), and an age of $t_{\star} \sim 12$ Myrs (Zuckerman et al. 2001). Note that the star's peak emission occurs at wavelength $\lambda=0.35 \mu \mathrm{m}$, yet the radius of the smallest bound grain is $R_{\text {min }} \simeq 3 \mu \mathrm{m}$ assuming the grains have a density of $\rho=1 \mathrm{gm} / \mathrm{cm}^{3}$ and a radiation pressure efficiency $Q_{r p}=0.53$ (Equation 6). So this system is in the geometric optics limit, and the lightscattering theory employed here is appropriate. Figure 11 of Golimowski et al. (2006) also shows that the disk's vertical distribution has a full width at half maximum (FWHM) that varies as FWHM $\propto 0.14 x \sim 2 h$, so the dust grains' inclinations are approximately $I=h / x \sim$ 0.07 radians.

The black curve in Figure 13 shows that the debris disk model can reproduce $\beta$ Pic's observed surface brightness when the dust-producing planetesimal disk is quite broad, with inner and outer radii $r_{i n}=75 \mathrm{AU}$ and $r_{\text {out }}=150 \mathrm{AU}$. This system has been evolved over the star's lifetime, $t_{\star}=12$ Myrs, so the system is still approaching equilibrium since $T_{e q}=20$ Myrs. The dust grains must also be rather asymmetric light scatters, with $g=0.67$ (assuming forward scattering), in order to account for the knee seen in the surface brightness profile at $x \sim 150 \mathrm{AU}$, consistent with Ahmic et al. (2009). The model also assumes that the dust grains are rather reflective, $Q_{s}=0.7$, which is the Bond albedo of Saturn's icy A and $\mathrm{B}$ rings at the observation wavelength (Porco et al. 2005). With these assumption in hand, fitting the simulated surface brightness to the disk's observed $B(x)$ requires the planetesimal disk's dust production rate to be $\dot{M}_{d}=1.7 \times 10^{15} \mathrm{gm} / \mathrm{sec}$. The total cross section of dust in the simulated debris disk is $A_{d}=1.9 \times 10^{20} \mathrm{~km}^{2}$, and the total mass of dust is $M_{d}=11$ lunar masses. Note that this mass is comparable to the 8 lunar masses that Holland et al. (1998) inferred from submillimeter observations of this disk, which supports the contention that the $\beta$ Pic dust grains are rather reflective.

The dust production rate inferred here is about $\dot{M}_{d} \simeq 9 \mathrm{M}_{\oplus} / \mathrm{Myr}$. For comparison, this rate is about 200 times higher than what Stern \& Colwell (1997) report for their model of the collisional erosion of our own Kuiper Belt. Note that $\beta$ Pic's dust production rate is quite considerable, for if it has held steady over the system's lifetime, then the unseen planetesimals orbiting $\beta$ Pic would have lost a total mass of $\dot{M}_{d} t_{\star} \sim 110$ earth-masses. 


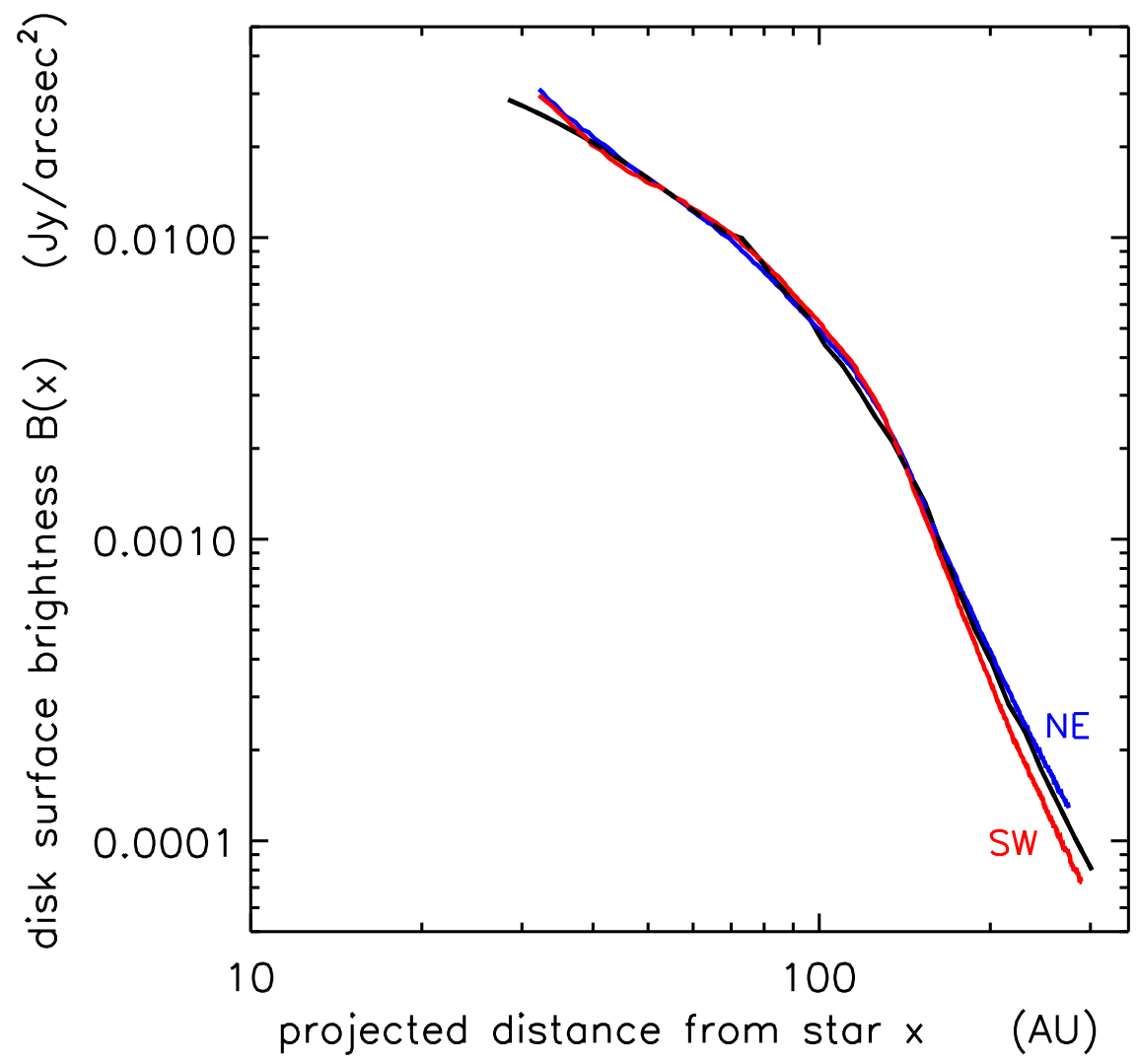

Fig. 13.- Red and blue curves give the surface brightness $B(x)$ of the edge-on disk orbiting $\beta$ Pictoris, extracted from the Hubble Space Telescope observations acquired by Golimowski et al. (2006) in the F606W filter, and plotted versus projected distance $x$ from the central star. The black dashed curve is for a model that adopts a $q=2.5$ size distribution for dust produced by a broad disk of planetesimals orbiting at $75<r<150$ AU from the central star; this system was evolved for $t=1.2 \times 10^{7} \mathrm{yrs}$, which is the age of the central star. The simulated dust grains' inclinations are $I=0.07 \mathrm{rad}=4.0^{\circ}$, their density is $\rho=1 \mathrm{gm} / \mathrm{cm}^{3}$, and they are strong with $Q^{\star}=10^{8} \mathrm{ergs} / \mathrm{gm}$. The dust grains also have a light scattering efficiency $Q_{s}=0.7$, a light-scattering asymmetry parameter $g=0.67$, and a radiation pressure efficiency $Q_{r p}=1-g Q_{s}=0.53$. The planetesimal disk's dust production rate is $\dot{M}_{d}=1.7 \times 10^{15} \mathrm{gm} / \mathrm{sec}$, so $N_{0}=3.6 \times 10^{34}$ and $T_{0}=1.5 \times 10^{5} \mathrm{yrs}$, and the disk's equilibrium timescale is $T_{e q}=2.0 \times 10^{7}$ yrs. 


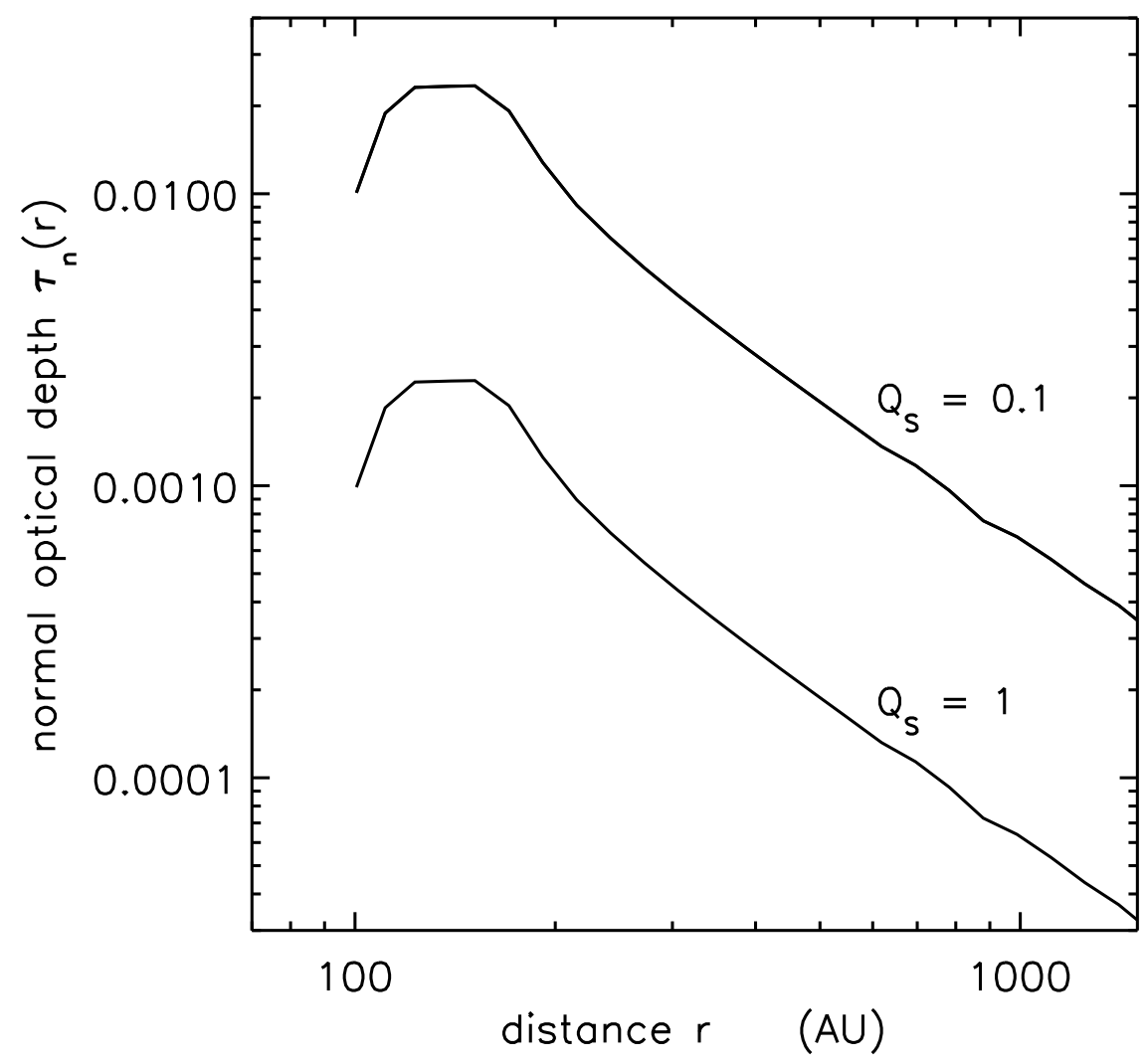

Fig. 14.- Normal optical depth $\tau_{n}(r)$ that results in the surface brightness profile shown in Figure 13, assuming $Q_{s}=0.1$ and $Q_{s}=1$. The dust production rate for the $Q_{s}=1$ model is 300 times smaller than the $Q_{s}=0.1$ model.

It should be noted that the planetesimal disk's inferred erosion rate is very sensitive to the dust grain's optical properties. Equation (31) shows that an edge-on disk's surface brightness is varies as $B \propto B_{0} N_{0} R_{\text {min }}^{2}$ where $B_{0} \propto Q_{s}, N_{0} \propto \dot{M}_{d}^{1 / 2} \rho^{2} Q_{r p}^{-5 / 2}$, and $R_{\min } \propto Q_{r p} / \rho$ (Equations 3, 6, 28a, and 30), which means that the inferred dust production rate varies as $\dot{M}_{d} \propto f_{Q}$ where $f_{Q}=Q_{r p} / Q_{s}^{2}$ and $Q_{r p}=1-g Q_{s}$. (Interestingly, the inferred dust production rate is insensitive to the assumed grain density $\rho$, which cancels out.) So if the dust grains are instead dark and have a scattering efficiency $Q_{s}=0.1$, then the radiation pressure efficiency is $Q_{r p} \simeq 1$ and $f_{Q} \simeq 100$, which means that the dust production rate must be $\sim 100$ faster if the dust grains are dark. In this case, $\beta$ Pic would have lost $\sim \dot{M}_{d} t_{\star} \sim 10^{4}$ earth-masses over its lifetime, which is implausible. Rather, it is more likely that the dust grains at $\beta$ Pic are bright.

The optical depth profile $\tau_{n}(r)$ that is inferred from $\beta$ Pic's observed surface brightness $B(x)$ is shown in Figure 14. Note that $B(x) \propto \int Q_{s} \tau_{n} d \ell$ where the integration is along the line of sight, so the disk's optical depth is uncertain by a factor of $Q_{s}$. The $Q_{s}=1$ curve 
obtained here is similar to the optical depth profile that Ahmic et al. (2009) inferred from the same dataset 3 . The range of possible values for the total dust mass $M_{d}$ and total dust cross section $A_{d}$ are $4.7 \leq M_{d} \leq 170$ lunar masses and $1.2 \times 10^{20} \leq A_{d} \leq 1.6 \times 10^{21} \mathrm{~km}^{2}$, with the lower values for a disk composed of bright $Q_{s}=1$ dust grains, and the higher values for dark $Q_{s}=0.1$ dust.

\section{1. sensitivity to parameters}

The following explores the sensitivity of the above results for $\beta$ Pic to the assumed model parameters.

First, the dust produced by the simulated planetesimal disk has a $q=2.5$ size distribution, so most of the just-produced dust grains' mass is in the largest grains that actually contribute little to the debris disk's optical depth. Consequently, the dust production rate $\dot{M}_{d}$ inferred above is somewhat sensitive to $\beta_{\text {min }}$, which is the size parameter of the largest grains that are allowed in the model. The equilibrium model shown in Figure 13 has $\beta_{\min }=0.065$ (which corresponds to a maximum grain radius $R_{\max }=23 \mu \mathrm{m}$ ), and it requires a dust production rate of $\dot{M}_{d}=1.7 \times 10^{15} \mathrm{gm} / \mathrm{sec}$ when the model is fit to the the disk's observed surface brightness profile assuming $Q_{s}=0.7$. However, cutting off the dust size distribution at $\beta_{\text {min }}=0.1$ and then optimizing the other parameters then results in a fit that is only marginally acceptable; that model has $R_{\max }=14 \mu \mathrm{m}$ and $\dot{M}_{d}=1.2 \times 10^{15} \mathrm{gm} / \mathrm{sec}$. Nonetheless, models having $\beta_{\min } \gtrsim 0.2$ are ruled out, because they are deficient in grains having sizes $R>6 \mu \mathrm{m}$ that would be confined to radial distances of $75 \lesssim r \lesssim 250 \mathrm{AU}$, and their absence from the model reduces the simulated disk's surface brightness at projected distances $x \sim r$.

Interestingly, the disk's surface brightness profile $B(x)$ is rather sensitive to the size distribution power law $q$. For instance, models having $q \leq 2.0$ do not reproduce the disk's observed surface density profile, because the model disk's inner $r \lesssim 150$ AU region is overdense with large dust grains that contribute too much surface brightness at $x \lesssim 150$ AU, while models having $q \geq 3.0$ are underdense in large grains and thus too dim there. Evidently, the dust being produced by the unseen planetesimals at $\beta$ Pic have a $q \simeq 2.5$ size distribution that is just a bit shallower than the canonical Dohnanyi $q=3.5$ size distribution. And because the observed dust size distribution is constrained to $2<q<3$, this translates into an uncertainty in the value of $\dot{M}_{d}$ quoted above is no more than a factor of 2 . Also, do keep in mind that the value for $q$ refers to the size distribution for dust production by the

\footnotetext{
${ }^{3}$ The Ahmic et al. (2009) work does not mention $Q_{s}$; we suspect that $Q_{s}=1$ was assumed there.
} 
planetesimal disk, and that the debris disk's resulting dust size distribution is actually much steeper due to collisions among dust grains (e.g., Figure 3).

This kind of modeling is also sensitive to the planetesimal disk's inner and outer radii. The midpoint of the knee at $x \simeq 110$ AU in $\beta$ Pic's surface brightness profile indicates that the planetesimal disk's midpoint is near $r \simeq 110$ AU (Figure 13), and best agreement with the observations is achieved when $r_{\text {in }} \simeq 75 \mathrm{AU}$ and $r_{\text {out }} \simeq 150 \mathrm{AU}$ i.e., when the disk has a radial aspect ratio of $r_{\text {in }} / r_{\text {out }} \simeq 0.5$. However, broader disks having $r_{\text {in }} / r_{\text {out }}<0.3$ are ruled out because the knee in their surface brightness profiles are too broad to fit the observations. Likewise, narrower planetesimal disks having $r_{\text {in }} / r_{\text {out }} \geq 0.8$ are ruled out because those models produce a prominent bump in the debris disk's surface brightness where $x=r_{i n}$, and such bumps are not seen in these optical observations. Although these surface brightness bumps are seen in infrared observations of $\beta$ Pic (Wahhaj et al. 2003; Telesco et al. 2005), their locations vary with the observing wavelength, which indicates that their interpretation likely requires modeling the disk's thermal emission as well.

We have also considered 'inside-out' erosion of the planetesimal disk, with $c=-2$, but the edge-on disk's surface brightness was rather similar to that generated by planetesimal disk suffering 'outside-in' erosion with $c=+2$, so $c=0$ is adopted in all models shown here. Also note that strong dust grains having $Q^{\star}=10^{8} \mathrm{ergs} / \mathrm{gm}$ are preferred over models that use weaker $Q^{\star} \leq 10^{7} \mathrm{ergs} / \mathrm{gm}$ dust grains. The larger grain strength increases the survival of the larger dust grains (see Figure 3) that are confined to the vicinity of the planetesimal disk at $75<r<150 \mathrm{AU}$. When $Q^{\star} \leq 10^{7} \mathrm{ergs} / \mathrm{gm}$, the lower abundance of large dust grains then results in a noticeable surface brightness deficit at $x \sim 100 \mathrm{AU}$.

\section{2. implications for planet formation}

If one adopts the plausible assumption that the $\beta$ Pic dust grains are bright $\left(Q_{s} \sim 0.7\right)$, then the mass of the planetesimal disk is likely well above $110 \mathrm{M}_{\oplus}$, which is sufficient to form about six Neptune-class planets. This planetesimal disk's considerable mass, plus its broad radial extent $(75 \lesssim r<150 \mathrm{AU})$, also provides several interesting constraints on the prospects for planet formation at $\beta$ Pic. Analogy with the Kuiper Belt, which resides at distances $\sim 30 \%$ beyond Neptune's orbit, suggests that there should be no Neptune-class giant planets orbiting well beyond $r \gtrsim 60$ AU from $\beta$ Pic, because such planets would have scattered away a portion of the inferred planetesimal disk and part of its dust disk. The fact that there are no such planets yet means that if planets are trying to form there via coreaccretion (e.g., a runaway accretion of planetesimals by a growing planetary embryo), then the timescale for this kind of assembly in the $75 \lesssim r<150$ AU zone must be longer than the 
system's $t_{\star} \sim 12$ Myr age. However, gravitational instability (GI) is a much faster planetformation mechanism, one that can also form giant planets at large stellarcentric distances (Durisen et al. 2007; Dodson-Robinson et al. 2009). However, GIs are only operative when the circumstellar disk is still rich in nebula gas, and the lifetime of a gas disk is at most a few Myrs. Consequently, the presence of $\beta$ Pic's broad and massive planetesimal disk also tells us that planet formation via GI did not happen in the $75 \lesssim r<150$ AU zone.

It can also be concluded that giant planets are unlikely to have formed interior to $r \sim 75$ AU and subsequently migrated or scattered deep into the $75 \lesssim r<150$ AU zone. For if that were to have happened at $\beta \mathrm{Pic}$, then the migrating/scattering planet would have accreted and/or scattered the planetesimal disk, causing the circumstellar dust to transition into a relic debris disk within a time $t_{\text {trans }} \sim 0.1 T_{e q} \sim 2$ Myrs. Consequently, the probability of an astronomer observing this system before its surface brightness transitioned into a relic $B(x) \propto x^{-5 / 2}$ profile is $t_{\text {trans }} / t_{\star} \sim 15 \%$. This finding is also consistent with Booth et al. (2009), who show that zero of 106 stars having circumstellar dust also appear to have experienced a Nice-model scattering of giant planets.

Of course, the Nice model was not intended to explain observations of circumstellar debris disks. However, our purpose here is to show how disk observations can be used to determine whether any particular Solar System's scenario might also be ongoing at other circumstellar disks, or if that scenario should be regarded as a rarity among the known population of star-disk systems. Indeed, it is a curiosity that $\beta$ Pic seems to be planetless at $r \gtrsim 75 \mathrm{AU}$ zone, despite having a substantial amount of planetesimal mass there. However $\beta$ Pic's heavy dust production rate, which is at least $\dot{M}_{d} \sim 9 \mathrm{M}_{\oplus} / \mathrm{Myr}$ (and may be much larger if the grains are darker than $Q_{s}=0.7$ ), suggests an alternate interpretation-that the planetesimal disk orbiting $\beta$ Pic is not a planet-producing disk, but is instead a planetesimaldestroying disk, due to a vigorous collisional grinding of planetesimals and a heavy mass-loss rate due to the blowout of dust by radiation pressure.

\section{3. $\quad$ Testing the assumptions}

This subsection examines the key assumptions that are employed here. Those assumptions are: (1) that collision fragments contribute little to the disk's optical depth, (2) the dusty disk is so tenuous that the dust grains are not shadowing each other, (3) that Poynting Robertson drag is insignificant, and (4) that the planetesimal disk's dust production is steady over time. A final subsection then comments on the fact that many debris disks are non-axisymmetric. 


\subsection{1. dust fragmentation}

When dust grains collide, they spawn smaller dust fragments, but their contribution to the debris disk's optical depth is ignored by this model. This is appropriate if the bulk of those fragments are sufficiently small and fast such that they are unbound and leave the system. It turns out that this assumption is quite reliable, and is confirmed from the model output. Begin with a single term in Equation (99), $\mathcal{R}_{i j k}=\alpha_{i j k} n_{i} n_{j} / T_{\text {out }}$, which is the rate at which the dust in the target streamline $i$ are destroyed due to collisions with the dust in streamline $j$ at the site $r=r_{i j k}$ where their orbits intersect. The resulting dust fragments are presumed to have a power-law size distribution $N_{f}=C R_{f}^{-q}$, which gives the number of dust fragments of radius $R_{f}$ produced by the destruction of a single target grain of radius $R_{i}$, with the coefficient $C$ determined by mass conservation. Equations (14 15) then provide the speed $v_{f}$ at which the fragments recoil from the collision, and their specific energy $E=\frac{1}{2} v_{f}^{2}-G M_{\star}\left(1-\beta_{f}\right) / r_{i j k}$ indicates whether the collision fragments having a size parameter $\beta_{f}$ will stay bound to the star or leave the system. The total rate at which the debris disk produces collision fragments of radius $R_{f}$ is $\tilde{\mathcal{R}}_{f}=\sum N_{f} \mathcal{R}_{i j k}$ where the sum proceeds over all sites in the disk $r_{i j k}$ where dust grains collide and produce bound fragments of radii $R_{f}$. This is the secondary dust production rate, and it is to be compared to the planetesimal disk's dust production rate $P_{f}$. The ratio $\tilde{\mathcal{R}}_{f} / P_{f}$ depends of the radius of the smallest possible fragment $R_{s}$. Also recall that $R_{\min }$ is the radius of the smallest bound dust grain that is produced in the planetesimal disk, so $R_{s}<R_{\min }$. In this instance, the above

algorithm finds that $\tilde{\mathcal{R}}_{f} \ll P_{f}$, which means that the rate at which collision are injecting second-generation dust fragments into the debris disk is negligible in comparison to the rate at which the planetesimal disk is injecting first-generation dust into the disk. For example, when $R_{s}<0.5 R_{\text {min }}$, this algorithm finds that $\tilde{\mathcal{R}}_{f} / P_{f}<1 \%$ when applied to the example model of Section 2.4.3. The ratio $\tilde{\mathcal{R}}_{f} / P_{f}$ is small because most of the collision fragments are small, unbound, and do not contribute to the debris disk, which justifies the model's neglect of dust fragmentation.

\subsection{2. shadowing}

If the disk is sufficiently dense, then dust grains can shadow each other and alter the disk's surface brightness profile $B(x)$. The calculations presented above all assumed that the dust grains are not shadowing each other, and that assumption is confirmed by examining the disk's radial optical depth $\tau_{r}$ (Weidenschilling 2010). Begin with a narrow annulus in the disk of radius $r$ and radial width $\Delta r$. The cross section of dust in that annulus is $\Delta A=$ $\tau_{n}(r) 2 \pi r \Delta r$ where $\tau_{n}(r)$ is the disk's normal optical depth. The annulus' vertical thickness 
is $2 I r$, so its optical depth along a radial line-of-sight is $\Delta \tau_{r}=\Delta A / 2 \pi r 2 \operatorname{Ir}=\tau_{n} \Delta r / 2 \operatorname{Ir}$. The total radial optical depth of dust interior to $r$ is then

$$
\tau_{r}(r)=\int_{0}^{r} \frac{\tau_{n}\left(r^{\prime}\right) d r^{\prime}}{2 I r^{\prime}}
$$

which is easily integrated numerically. When this quantity is not small, then the dust grains at $r$ are shadowed by interior dust, which reduces the stellar illumination there by a factor $e^{-\tau_{r}}$. But if $\tau_{r} \ll 1$ then the dust grains are fully illuminated.

Most of the debris disk models described in the previous sections have $\tau_{r}<10^{-3}$. The two exceptions are the heavy dust-producing models, such as those having $\dot{M}_{d}=10^{15} \mathrm{gm} / \mathrm{sec}$ that have $\tau_{r} \sim 0.01$ (Figures 4, 6, and 9), while the $\beta$ Pic model that considers dark $Q_{s}=0.1$ dust grains has $\tau_{r} \sim 0.04$. So in summary, shadowing by dust grains is not significant for the debris-disk models considered here.

\subsubsection{Poynting Robertson drag}

Poynting-Robertson (PR) drag is a weak force that causes the orbits of small dust grains to slowly decay. The following shows that this orbital decay is negligible for the dust in the $\beta$ Pic debris disk.

PR drag is the acceleration that results when a moving grain absorbs and/or scatters stellar photons. Because a grain's motion also results in a slight 'headwind' of photons, the transfer of momentum, from the photons to the grain, causes its orbit to decay. The acceleration on a dust grain due to PR drag is (Burns et al. 1979)

$$
\mathbf{a}_{P R}=-\frac{\beta G M_{\star}}{r^{2}}\left(\frac{2 v_{r}}{c} \hat{\mathbf{r}}+\frac{v_{\theta}}{c} \hat{\theta}\right)
$$

where $v_{r}$ and $v_{\theta}$ are the grain's radial and tangential velocities (Equations 13) and $\hat{\mathbf{r}}, \hat{\theta}$ are unit vectors in a polar coordinate system. Inserting this into the Lagrange planetary equations and time-averaging those equations over an orbit then provides the rates at which an orbiting dust grain's semimajor axis $a$ and eccentricity $e$ decay due to PR drag,

$$
\begin{aligned}
\dot{a} & =-\frac{2 \beta G M_{\star}}{a c} \frac{1+\frac{3}{2} e^{2}}{\left(1-e^{2}\right)^{3 / 2}} \\
\text { and } \quad \dot{e} & =-\frac{5 \beta G M_{\star} e}{2 \sqrt{1-e^{2}} a^{2} c}
\end{aligned}
$$

(Wyatt \& Whipple 1950; Burns et al. 1979). The timescales associated with this orbit decay 
are

$$
\begin{aligned}
T_{a} & =\frac{a}{|\dot{a}|}=\frac{\left(1-e^{2}\right)^{3 / 2}}{\beta\left(1+\frac{3}{2} e^{2}\right)}\left(\frac{a}{r_{\text {out }}}\right)^{2} T_{P R} \\
\text { and } T_{e} & =\frac{e}{|\dot{e}|}=\frac{4 \sqrt{1-e^{2}}}{5 \beta}\left(\frac{a}{r_{\text {out }}}\right)^{2} T_{P R}
\end{aligned}
$$

where the constant

$$
T_{P R}=\frac{r_{\text {out }}^{2} c}{2 G M_{\star}}=2.0 \times 10^{6}\left(\frac{M_{\star}}{M_{\odot}}\right)^{-1}\left(\frac{r_{\text {out }}}{50 \mathrm{AU}}\right)^{2} \mathrm{yrs} .
$$

Inserting Equations (15) and (7) into the above shows that these timescales are simple functions of grain size $\beta$ or $R$. If these orbit decay timescales are long compared to a dust grains' collisional lifetime $T_{c}$, then it is appropriate to ignore PR drag.

These orbit decay timescale are plotted in Figure 4 for the example debris disk that is generated by a planetesimal ring of radius $r_{\text {out }}=50 \mathrm{AU}$ (see Sections 2.4.3 -2.4.5 and Figures 2 11). All of these curves diverge for small grains that have radii $R \simeq R_{\text {min }}$ and size

parameters $\beta \simeq \frac{1}{2}$. Note that for most grain sizes $T_{c}(R) \ll T_{a}(R)$, which means that the effects of PR drag are negligible over the lifetime of grains that are only slightly larger than $R_{\text {min }}$. Figure 4 also shows shows that PR drag is only significant when the system's dust production rate $\dot{M}_{d}$ is sufficiently low, which can make $T_{c} \gtrsim T_{a}$ large, but only for these small $R \simeq R_{\text {min }}$ grains. But this only occurs when $\dot{M}_{d} \lesssim 10^{11} \mathrm{gm} / \mathrm{sec}$, for the scenario considered in Figure 4. A similar result was obtained by Wyatt (2005), who showed that PR drag is only significant when the debris disk is sufficiently tenuous. And finally, note that PR drag is completely negligible $\left(T_{c} \ll T_{a}\right)$ for grains of all sizes in the $\beta$ Pic debris disk, due to its very vigorous dust production rate and the grains' short collisional lifetimes.

\subsection{4. dust production over time}

The model employed here assumes that the planetesimal disk's dust production rate is steady over time. But this assumption might seem debatable, because the collisional erosion that drives dust production will ultimately decrease the planetesimal disk's mass over time. But keep in mind that accretion within the planetesimal disk tends to produce larger bodies whose gravity can stir-up the planetesimal disk, and that can instead increase the disk's dust production rate. Further, simulations of the collisional/accretional evolution of a planetesimal disk shows that the outcomes are very sensitive to the planetesimal disk's initial conditions that are poorly known (Weidenschilling 2010). Due to this uncertainty, and that any rigorous treatment of the planetesimal disk's evolution goes well beyond the 
intended scope of this study, this model makes the simplest possible assumption, that the planetesimal disk's dust production rate is steady over time. This assumption is also justified by the collisional evolution models of Stern \& Colwell (1997) and Kenyon (2002), who obtain a slow erosion timescale of $\sim 500$ Myrs for a Kuiper Belt orbiting at $r \sim 40$ AU. Because the timescale over which the erosion-rate varies is likely longer than $\beta$ Pic's equilibrium timescale $\left(T_{e q} \sim 20\right.$ Myrs, Section 3) , the debris disk is expected to remain in quasi-static equilibrium as it adjusts to any slow change in the dust production rate. Consequently, the debris-disk mass and dust production rate inferred here are expected to be reliable.

Also note that $\beta$ Pic's dust production rate is considerable, at least $\dot{M}_{d} \sim 9 M_{\oplus} /$ Myr. Consequently, steady dust production over the age of the system $t_{\star}$ also requires a large reservoir of planetesimal mass $M_{p}$, with $M_{p} \gg \dot{M}_{d} t_{\star} \sim 110 M_{\oplus}$, i.e., the mass in $\beta$ Pic's Kuiper Belt at $75 \lesssim r \lesssim 150$ AU must be well in excess of 110 earth masses. Alternatively, if $\beta$ Pic's Kuiper Belt does not satisfy $M_{p} \gg 110 M_{\oplus}$, then collisions among planetesimals plus blowout by radiation pressure is going to grind down this extra-solar Kuiper Belt in a few $\times t_{\star} \sim 10$ 's of Myr. In this case the assumption of steady dust production does not hold, but it does mean that dust production was probably more vigorous in the past. Regardless, $\beta$ Pic's planetesimal disk is or was very massive.

\subsection{5. disk asymmetry}

Many edge-on debris disks are lopsided, with one ansa substantially brighter than the other; examples include $\beta$ Pic (Heap et al. 2000), AU Mic (Krist et al. 2005), and HD 15115 (Kalas et al. 2007). It may be that circumstellar debris disks are routinely non-axisymmetric. Once possible explanation for a disk's asymmetry is a recent dust-producing collision in the planetesimal disk. Recall that the orbits of all the dust grains produced in a single collision have their longitudes of periapse $\tilde{\omega}$ aligned (Section 2.1). Because dust produced in a collision tend to loiter at their apoapse, the debris disk will have an excess of dust at longitudes $\theta=\tilde{\omega}+\pi$. So if that collision was vigorous enough to produce a substantial amount of dust, then the disk will be non-axisymmetric, and may appear lopsided when viewed edge on.

Another possible explanation for a disk's asymmetry is described in Hahn (2009), who showed that if the circumstellar dust is produced by an eccentric planetesimal ring, then the smaller dust grains produced at the ring's periapse are less bound to the star due to their higher orbital velocities there. Consequently, there will be fewer small and marginally bound grains in the direction of the ring's apoapse, which will make that part of the debris disk dimmer than the periapse side that also has an excess of small grains. This is a particularly interesting scenario since it also implies that an extra-solar planet is likely present in order 
to maintain the planetesimal ring's forced eccentricity.

Both of these scenarios will be explored in a followup study using a more advanced version of the debris-disk model that will be generalized to handle the collisional evolution of a non-axisymmetric debris disk.

Lastly, it should be noted that this model also ignores the planetesimals' free eccentricity $e_{f}$ that is associated with the random part of their noncircular motions. Equations (13-14) in Thébault et al. (2003) show that the planetesimals' random motions tends to blur the simple relationship between a dust grain's orbit and its size parameter (e.g., Equations 5 and (7). However this effect will be small when the planetesimals eccentricity $e_{f}$ is small, which in fact is a requirement in order for planetesimals to have formed in the first place.

\section{Summary and Conclusions}

A numerical model for a circumstellar debris disk is developed and applied to observations of $\beta$ Pictoris. The model accounts for dust production by colliding planetesimals and dust destruction due to collisions among grains. These rates for dust production and collisional destruction also provide a rate equation whose solution gives the dust abundance over time and as a function of grain size. That solution also provides the debris disk's grain size distribution, which is steepened substantially by collisions among dust grains. These calculations also give the dust grains' collisional lifetime $T_{c}(R)$, which depends on the planetesimal disk's dust production rate $\dot{M}_{d}$ and grain radius $R$.

Scaling laws are derived, and it is shown that the dust abundance $N$ in the debris disk varies as $N \propto \dot{M}_{d}^{1 / 2}$ once the disk has settled into collisional equilibrium, and that dust lifetimes vary as $T_{c} \propto \dot{M}_{d}^{-1 / 2}$. It is also shown that the radial drift of dust grains due to PR drag is unimportant provided the dust production rate is sufficiently high so that the grains' collisional lifetimes are short compared to the drift timescale. The model also recovers the results of Strubbe \& Chiang (2006), who showed that the debris disk's optical depth varies as $\tau_{n}(r) \propto r^{-3 / 2}$ when in equilibrium, and that an edge-on disk's surface brightness varies as $B(x) \propto x^{-7 / 2}$, where $r$ and $x$ are the radial and projected distances from the central star. Note though that those optical depth and surface brightness profiles will be steeper if the disk is young and not yet in collisional equilibrium. Alternatively, those profiles will be shallower if dust production has ceased in the past, which might occur if planets form in (or migrate or scatter into) the planetesimal disk and cause its dynamical depletion.

It is also shown that these quantities are not very sensitive to the dust grains' strength $Q^{\star}$ when $Q^{\star}<10^{8} \mathrm{ergs} / \mathrm{gm}$. However, an edge-on disk's surface brightness profile is quite 
sensitive to the dust grain's light scattering asymmetry parameter $g$, and disks like the ones at $\beta$ Pic and AU Mic having a 'knee' in their surface brightness indicates that the dust are very asymmetric light scatters, with $|g| \gtrsim 0.7$.

The model's principal dynamical parameters are the planetesimal disk's radius and its dust production rate, so a comparison to observations then yields estimates of or else limits on these important cosmogonic quantities. For instance, fitting the model to optical HST observations of $\beta$ Pic shows good agreement with the disk's observed surface brightness profile when the unseen dust-producing planetesimal disk there is quite broad, extending over $75 \lesssim r \lesssim 150$ AU. This disk's dust production rate is also quite heavy, $\dot{M}_{d} \sim 9$ $\mathrm{M}_{\oplus} / \mathrm{Myr}$, if it is assumed that the dust grains are bright like Saturn's $\operatorname{rings}\left(Q_{s}=0.7\right)$. In this case, the total cross section of dust in the debris disk is $A_{d}=1.9 \times 10^{20} \mathrm{~km}^{2}$, and the inferred mass there is $M_{d}=11$ lunar masses. Note that this mass is comparable to that previously inferred from submillimeter observations of this disk (Holland et al. 1998). Also, it is unlikely that the dust grains at $\beta$ Pic are dark (i.e., with $Q_{s}<0.1$ ), since that would require the planetesimal disk to grind away at a rate that is implausible. Indeed, the mass-loss rates inferred here are so heavy as to suggest that the $r \gtrsim 75$ AU zone at $\beta$ Pic might be a region of planetesimal destruction due to collisional grinding, rather than a site of ongoing planet formation.

The model developed here is called ddisk, which is an easy-to-use IDL script that others might wish to use as they diagnose their observations of circumstellar debris disks. This code is available for download at http://gemelli.spacescience.org/ hahnjm/software.html.

\section{Acknowledgments}

Support for this work was provided by NASA through grant number HST-AR-11754.01A from the SPACE TELESCOPE SCIENCE INSTITUTE, which is operated by the Association of Universities for Research in Astronomy, Inc., under NASA contract NAS5-26555. JMH also thanks an anonymous reviewer for several helpful suggestions.

\section{REFERENCES}

Ahmic, M., Croll, B., \& Artymowicz, P. 2009, ApJ, 705, 529

Booth, M., Wyatt, M. C., Morbidelli, A., Moro-Martín, A., \& Levison, H. F. 2009, MNRAS, 399,385

Burns, J. A., Lamy, P. L., \& Soter, S. 1979, Icarus, 40, 1 
Crifo, F., Vidal-Madjar, A., Lallement, R., Ferlet, R., \& Gerbaldi, M. 1997, A\&A, 320, L29

Dodson-Robinson, S. E., Veras, D., Ford, E. B., \& Beichman, C. A. 2009, ApJ, 707, 79

Dohnanyi, J. W. 1969, J. Geophys. Res., 74, 2531

Durda, D. D. \& Dermott, S. F. 1997, Icarus, 130, 140

Durisen, R. H., Boss, A. P., Mayer, L., Nelson, A. F., Quinn, T., \& Rice, W. K. M. 2007, Protostars and Planets V, 607

Golimowski, D. A., Ardila, D. R., Krist, J. E., Clampin, M., Ford, H. C., Illingworth, G. D., Bartko, F., Benítez, N., Blakeslee, J. P., Bouwens, R. J., Bradley, L. D., Broadhurst, T. J., Brown, R. A., Burrows, C. J., Cheng, E. S., Cross, N. J. G., Demarco, R., Feldman, P. D., Franx, M., Goto, T., Gronwall, C., Hartig, G. F., Holden, B. P., Homeier, N. L., Infante, L., Jee, M. J., Kimble, R. A., Lesser, M. P., Martel, A. R., Mei, S., Menanteau, F., Meurer, G. R., Miley, G. K., Motta, V., Postman, M., Rosati, P., Sirianni, M., Sparks, W. B., Tran, H. D., Tsvetanov, Z. I., White, R. L., Zheng, W., \& Zirm, A. W. 2006, AJ, 131, 3109

Gomes, R., Levison, H. F., Tsiganis, K., \& Morbidelli, A. 2005, Nature, 435, 466

Gomes, R. S., Morbidelli, A., \& Levison, H. F. 2004, Icarus, 170, 492

Hahn, J. M. 2009, in AAS/Division of Dynamical Astronomy Meeting, Vol. 40, AAS/Division of Dynamical Astronomy Meeting, \#06.12-+

Heap, S. R., Lindler, D. J., Lanz, T. M., Cornett, R. H., Hubeny, I., Maran, S. P., \& Woodgate, B. 2000, ApJ, 539, 435

Holland, W. S., Greaves, J. S., Zuckerman, B., Webb, R. A., McCarthy, C., Coulson, I. M., Walther, D. M., Dent, W. R. F., Gear, W. K., \& Robson, I. 1998, Nature, 392, 788

Holsapple, K., Giblin, I., Housen, K., Nakamura, A., \& Ryan, E. 2002, Asteroids III, 443

Holsapple, K. A. 1994, Planet. Space Sci., 42, 1067

Kalas, P., Fitzgerald, M. P., \& Graham, J. R. 2007, ApJ, 661, L85

Kenyon, S. J. 2002, PASP, 114, 265

Krist, J. E., Ardila, D. R., Golimowski, D. A., Clampin, M., Ford, H. C., Illingworth, G. D., Hartig, G. F., Bartko, F., Benítez, N., Blakeslee, J. P., Bouwens, R. J., Bradley, L. D., Broadhurst, T. J., Brown, R. A., Burrows, C. J., Cheng, E. S., Cross, N. J. G., 
Demarco, R., Feldman, P. D., Franx, M., Goto, T., Gronwall, C., Holden, B., Homeier, N., Infante, L., Kimble, R. A., Lesser, M. P., Martel, A. R., Mei, S., Menanteau, F., Meurer, G. R., Miley, G. K., Motta, V., Postman, M., Rosati, P., Sirianni, M., Sparks, W. B., Tran, H. D., Tsvetanov, Z. I., White, R. L., \& Zheng, W. 2005, AJ, 129, 1008

Krivov, A. V., Löhne, T., \& Sremčević, M. 2006, A\&A, 455, 509

Lamy, P. L. \& Perrin, J. 1986, A\&A, 163, 269

Lester, T. P., McCall, M. L., \& Tatum, J. B. 1979, JRASC, 73, 233

Levison, H. F., Morbidelli, A., Vanlaerhoven, C., Gomes, R., \& Tsiganis, K. 2008, Icarus, 196,258

Löhne, T., Krivov, A. V., \& Rodmann, J. 2008, ApJ, 673, 1123

Meyer, M. R., Backman, D. E., Weinberger, A. J., \& Wyatt, M. C. 2007, in Protostars and Planets V, ed. B. Reipurth, D. Jewitt, \& K. Keil, 573-588

Porco, C. C., Baker, E., Barbara, J., Beurle, K., Brahic, A., Burns, J. A., Charnoz, S., Cooper, N., Dawson, D. D., Del Genio, A. D., Denk, T., Dones, L., Dyudina, U., Evans, M. W., Giese, B., Grazier, K., Helfenstein, P., Ingersoll, A. P., Jacobson, R. A., Johnson, T. V., McEwen, A., Murray, C. D., Neukum, G., Owen, W. M., Perry, J., Roatsch, T., Spitale, J., Squyres, S., Thomas, P., Tiscareno, M., Turtle, E., Vasavada, A. R., Veverka, J., Wagner, R., \& West, R. 2005, Science, 307, 1226

Stern, S. A. \& Colwell, J. E. 1997, ApJ, 490, 879

Strubbe, L. E. \& Chiang, E. I. 2006, ApJ, 648, 652

Telesco, C. M., Fisher, R. S., Wyatt, M. C., Dermott, S. F., Kehoe, T. J. J., Novotny, S., Mariñas, N., Radomski, J. T., Packham, C., De Buizer, J., \& Hayward, T. L. 2005, Nature, 433, 133

Thébault, P., Augereau, J. C., \& Beust, H. 2003, A\&A, 408, 775

Wahhaj, Z., Koerner, D. W., Ressler, M. E., Werner, M. W., Backman, D. E., \& Sargent, A. I. 2003, ApJ, 584, L27

Weidenschilling, S. J. 2010, submitted to ApJ

Wyatt, M. C. 2005, A\&A, 433, 1007 
Wyatt, M. C., Smith, R., Greaves, J. S., Beichman, C. A., Bryden, G., \& Lisse, C. M. 2007a, ApJ, 658, 569

Wyatt, M. C., Smith, R., Su, K. Y. L., Rieke, G. H., Greaves, J. S., Beichman, C. A., \& Bryden, G. 2007b, ApJ, 663, 365

Wyatt, S. P. \& Whipple, F. L. 1950, ApJ, 111, 134

Zuckerman, B., Song, I., Bessell, M. S., \& Webb, R. A. 2001, ApJ, 562, L87 